\title{
ESTIMATION OF A SEMIPARAMETRIC IGARCH(1,1) MODEL ${ }^{*}$
}

\author{
Woocheol Kim ${ }^{\dagger}$ \\ (Korea Institute of Public Finance)
}

\author{
Oliver Linton ${ }^{\ddagger}$ \\ (London School of Economics and Universidad Carlos \\ III de Madrid)
}

DP No: EM 2009539

October 2009

The Suntory Centre

Suntory and Toyota International Centres for

Economics and Related Disciplines

London School of Economics and Political Science Houghton Street

London WC2A $2 A E$

Tel: 02079556674

\footnotetext{
* We would like to thank Xiaohong Chen, Joel Horowitz, and Eric Renault for interesting discussions. We thank also Jean-Pierre Florens and two referees for helpful comments.

${ }^{\dagger}$ Korea Institute of Public Finance, 79-6 Garak-Dong, Songpa-Gu, Seoul, Republic of Korea, 138774; email: wkim@kipf.re.kr

₹ Department of Economics, London School of Economics, Houghton Street, London WC2A 2AE, United Kingdom. E-mail address: o.linton@Ise.ac.uk. Thanks to the ESRC and Leverhulme foundations for financial support. This paper was partly written while I was a Universidad Carlos III de Madrid-Banco Santander Chair of Excellence, and I thank them for financial support.
} 


\begin{abstract}
We propose a semiparametric IGARCH model that allows for persistence in variance but also allows for more flexible functional form. We assume that the difference of the squared process is weakly stationary. We propose an estimation strategy based on the nonparametric instrumental variable method. We establish the rate of convergence of our estimator.
\end{abstract}

Key words and phrases: Inverse Problem; Instrumental Variable; IGARCH; Kernel Estimation; Nonparametric regression.

Journal of Economic Literature Classification: C14

(C) The authors. All rights reserved. Short sections of text, not to exceed two paragraphs, may be quoted without explicit permission provided that full credit, including (c notice, is given to the source. 


\section{Introduction}

A number of authors have found parameter estimates in $\operatorname{GARCH}(1,1)$ models close to the unit root region, and have proposed using the integrated GARCH or IGARCH process which imposes this restriction, see for example Engle and Bollerslev (1986). The exponentially weighted moving average model (EWMA) (aka 'JP Morgan' model), which is the special case in which the intercept is set to zero, is in wide use by practitioners. The IGARCH process although it does not possess a finite (limiting) variance can be strongly stationary, see Nelson (1990). In fact, IGARCH processes can also be geometrically strong mixing, see Meitz and Saikonnen (2004).

We propose a semiparametric extension of the IGARCH model. Our model nests the standard IGARCH $(1,1)$ model, but it allows more flexibility in functional form. It extends the recent model of Linton and Mammen (2005) to the case where the unconditional variance of the process does not exist. We propose an estimation method that involves solving a type one integral equation with estimated operator, Carrasco, Florens and Renault (2006). We establish the rate of uniform convergence of the nonparametric part of our model and the consistency of the parametric part.

All proofs are given in the appendix.

\section{The Model}

We suppose throughout that $\left\{y_{t}\right\}_{t=-\infty}^{\infty}$ is an observed strongly stationary process. We suppose that

$$
\begin{aligned}
y_{t} & =\sigma_{t} \varepsilon_{t} \\
\sigma_{t}^{2} & =\beta \sigma_{t-1}^{2}+(1-\beta) y_{t-1}^{2}+m\left(y_{t-1}\right)
\end{aligned}
$$

where $\varepsilon_{t}$ and $\varepsilon_{t}^{2}-1$ are martingale difference sequences i.e., $E\left(\varepsilon_{t} \mid \mathcal{F}_{t-1}\right)=0$ and $E\left(\varepsilon_{t}^{2}-1 \mid \mathcal{F}_{t-1}\right)=0$, where $\mathcal{F}_{t}$ contains all information upto current period, and $m(\cdot)$ is an unknown function. When $m(y)=\omega$ for some constant $\omega$, the above model reduces to a standard parametric $\operatorname{IGARCH}(1,1)$ model and when $\omega=0$ it is the EWMA. If $m(y)=\delta y^{2}+\omega$, then the process is an 'explosive' GARCH process, strictly stationary for some range of $\delta \geq 0$. In general, we allow the nonparametric function $m(\cdot)$ to take a flexible form, as long as it satisfies some regularity condition including smoothness, a nonnegativity constraint $(m(\cdot) \geq 0)$, and some additional conditions guaranteeing strong stationarity of $y_{t}$. The nonparametric term is introduced to correct possible misspecification with a quadratic growth function of news impact on volatility.

Defining the martingale difference sequence $\eta_{t}=\sigma_{t}^{2}\left[\varepsilon_{t}^{2}-1\right]$, we write the squared returns as 
$y_{t}^{2}=\sigma_{t}^{2}+\eta_{t}$. By plugging into (1), we get

$$
\begin{aligned}
\Delta y_{t}^{2}=y_{t}^{2}-y_{t-1}^{2} & =m\left(y_{t-1}\right)+(1-\beta) \eta_{t-1}+\eta_{t}-\eta_{t-1} \\
& =m\left(y_{t-1}\right)+\eta_{t}-\beta \eta_{t-1}
\end{aligned}
$$

The squared returns $\left\{y_{t}^{2}\right\}$ is an integrated process with a functional drift term, $m(\cdot)$, and moving average error term. If $m(\cdot) \leq c$ (with $c$ not so large), the model is likely to show a similar dynamics to the standard IGARCH process.

For our theoretical development, it is important that $\left\{\Delta y_{t}^{2}\right\}$ satisfies weak stationarity even when the process $\left\{y_{t}^{2}\right\}$ does not. In the context of linear time series models this is a property that is quite common, but in the current context it is not obviously possible. Harvey, Ruiz, and Shephard (1994) say that (in the case where $\varepsilon_{t}$ is standard normal) " $\left\{\Delta y_{t}^{2}\right\}$ is stationary and has an ACF like that of an MA(1) process." Their argument seems to be based on the fact that the innovation process $\eta_{t}$ appears to be a martingale difference sequence. Although it appears that $E\left[\eta_{t} \mid \mathcal{F}_{t-1}\right]=0$, this definition only makes sense if $E\left[\left|\eta_{t}\right|\right]<\infty$ or $\lim _{t \rightarrow \infty} E\left[\left|\eta_{t}\right| \mid \mathcal{F}_{0}\right]<\infty$ [Hall and Heyde (1980)]. In fact, these conditions do not hold. Therefore, one cannot conclude anything about weak stationarity. In any case, weak stationarity of $\Delta y_{t}^{2}$ requires both its mean and variance to exist, which would require that $\lim _{t \rightarrow \infty} E\left[\eta_{t}^{2} \mid \mathcal{F}_{0}\right]<\infty$. In fact, $\left\{\Delta y_{t}^{2}\right\}$ is not weakly stationary in the Gaussian strong IGARCH. The cause of this counterintuitive (from the point of view of linear processes) behaviour is due to the i.i.d. innovation. The following example shows that when the innovations are not i.i.d. one can have $\left\{\Delta y_{t}^{2}\right\}$ weak stationarity [or at least have finite first moment] even when $\left\{y_{t}\right\}$ is not weakly stationary.

EXAMPLE: Consider a semi-strong IGARCH model, i.e., (1) with $m(y)=\omega$ and

$$
\varepsilon_{t}=\operatorname{sign}\left(z_{t}\right)\left\{1+\frac{u_{t}^{2}-1}{1+\sigma_{t}^{2}}\right\}^{1 / 2},
$$

where $z_{t}, u_{t}$ are i.i.d. and mutually independent random variables with $E \operatorname{sign}\left(z_{t}\right)=0$ and $E u_{t}^{2}=1$. It follows that $\varepsilon_{t}$ and $\varepsilon_{t}^{2}-1$ are martingale difference sequences and thereby are consistent with (1). Furthermore, $\eta_{t}=\left(u_{t}^{2}-1\right)\left(\sigma_{t}^{2} /\left(1+\sigma_{t}^{2}\right)\right)$ which satisfies $E\left(\left|\eta_{t}\right|\right)<\infty$, and so we have $E\left(\left|\Delta y_{t}^{2}\right|\right)<\infty$. However, $E\left(\sigma_{t}^{2}\right)=E\left(y_{t}^{2}\right)=\infty$. If $z_{t}$ is symmetric about zero, then $\varepsilon_{t}$ is also symmetrically distributed both conditionally and unconditionally. Provided $u_{t}$ has finite fourth moment then $E\left(\left[\Delta y_{t}^{2}\right]^{2}\right)<\infty$.

In the sequel we shall assume that the process $\left\{\Delta y_{t}^{2}\right\}$ is weakly stationary. This has strong and testable empirical implications and we investigate whether this is a reasonable assumption in some common datasets below.

An interesting feature of our semiparametric IGARCH model is that the nonlinear correction term $m$ can be identified independently of $\beta$. Significant estimates of $m$ are related directly to 
misspecification of IGARCH. Also, by means of nonparametric function $m$, (1) can nest both GARCH and IGARCH as a special case. This model is related to recent work of Linton and Mammen (2005) who considered the case with $\sigma_{t}^{2}=\beta \sigma_{t-1}^{2}+m\left(y_{t-1}\right)$ and $E\left(y_{t}^{2}\right)<\infty$. The estimation strategy there involved solving a Type 2 integral equation and was simpler to analyze. The estimation strategy we develop here can be used in their model, but yields poorer rates of convergence.

\section{Identification}

Let $f_{0}(\cdot)$ be the marginal density function of $y_{t}$, and denote the joint density function of $\left(y_{t-1}, y_{t-k}\right)$ and $\left(\Delta y_{t}^{2}, y_{t-k}\right)$ by $f_{k}(\cdot, \cdot)$ and $f_{k}^{\Delta}(\cdot, \cdot)$, respectively, for suitable $k$. Letting $\nu_{t}=\eta_{t}-\beta \eta_{t-1}$, we write (1) as

$$
\Delta y_{t}^{2}=m\left(y_{t-1}\right)+\nu_{t}
$$

where $E\left(\nu_{t} \mid y_{t-1}\right) \neq 0$ but $E\left(\nu_{t} \mid y_{t-k}\right)=0$ for $k \geq 2$; (3) is an example of nonparametric structural models with infinite number of instruments. By the finite moment condition $\left(E\left(\left|\Delta y_{t}^{2}\right|\right)<\infty\right)$ and the law of iterated expectations, we obtain, from taking conditional expectations of (3),

$$
E\left[m\left(y_{t-1}\right) \mid y_{t-k}=w\right]=E\left[\Delta y_{t}^{2} \mid y_{t-k}=w\right], \quad \text { for all } k \geq 2 .
$$

This can be viewed as an integral equation of the first kind with solution $m($.$) . It is convenient to$ multiply both sides of the equation by the marginal density of $y_{t}$ evaluated at $w$, which preserves the equation but makes the analysis a bit simpler. Define the linear operator $\mathcal{T}_{k}: L^{2}(\mathcal{Y}) \rightarrow L^{2}(\mathcal{Y})$ by

$$
\mathcal{T}_{k} m(w)=\int m(x) f_{k}(x, w) d x=E\left[m\left(y_{t-1}\right) \mid y_{t-k}=w\right] f_{0}(w),
$$

and a reduced form function $h_{k}(\cdot)$ by

$$
h_{k}(w)=\int z f_{k}^{\Delta}(z, w) d z=E\left(\Delta y_{t}^{2} \mid y_{t-k}=w\right) f_{0}(w),
$$

where $f_{0}(\cdot)$ is the marginal density function of $y_{t}$. Then, $m(\cdot)$ satisfies the equation

$$
\mathcal{T}_{k} m(w)=h_{k}(w)
$$

for all $k \geq 2$. The solution to the integral equation is unique (if it exists), if and only if, for some $k \geq 2, \mathcal{T}_{k}$ is one-to-one, or equivalently, the conditional distribution of $y_{t-1}$, given $y_{t-k}$, is statistically complete in the sense that $E\left[m\left(y_{t-1}\right) \mid y_{t-k}\right]=0$, a.s., only for $m \equiv 0$. A sufficient condition for completeness is that the conditional CDF, $F_{1 \mid k}(\cdot \mid \cdot)$, is a member of an exponential family satisfying certain regularity conditions given in Newey and Powell (2003). In Blundell, Chen, and Kristensen (2003), an alternative but weaker condition is suggested for bounded completeness. Since we implicitly 
assume that $m$ is uniformly bounded, the latter kind of completeness may be more relevant for our model. However, considering that the process $\left\{y_{t}\right\}$ defined by (1) does not possess a finite second moment, the aforementioned approaches cannot be applied here. Our identification result below is based on the approach of Kim (2003). We could establish identification from (5) for any single $k$ or from a finite set of such moment equations. However, motivated by the work of Linton and Mammen (2005) we combine all the equations (5) into a single equation using a weighting sequence and establish identification for this weighted equation.

Let $f_{\lambda}(x, w)=\sum_{k=2}^{\infty} \lambda_{k} f_{k}(x, w)$ and $h_{\lambda}(w)=\sum_{k=2}^{\infty} \lambda_{k} h_{k}(w)$, for $\lambda_{k} \geq 0$ with $\sum_{k=2}^{\infty} \lambda_{k}=1$. This includes the case where $\lambda_{k}=1$ and $\lambda_{j}=0$ for all $j \neq k$, and it also includes the case where $\lambda_{k}=\beta^{k}$, which turned out to be the optimal equation in Linton and Mammen (2005). Define $\mathcal{T}_{\lambda}: L^{2}(\mathcal{Y}) \rightarrow$ $L^{2}(\mathcal{Y})$ to be a linear operator such that $\mathcal{T}_{\lambda}=\sum_{k=2}^{\infty} \lambda_{k} \mathcal{T}_{k}$, then we have $\mathcal{T}_{\lambda} m(w)=h_{\lambda}(w)$. We will define identifiability of $m$ in the context of this equation.

Definition 3.1 The true function $m_{0}(\cdot)$ is identifiable if the solution to the following integral equation

$$
\mathcal{T}_{\lambda} m(w)=\int m(x) f_{\lambda}(x, w) d x=h_{\lambda}(w)
$$

is unique or equivalently if $\mathcal{T}_{\lambda}: L^{2}(\mathcal{Y}) \rightarrow L^{2}(\mathcal{Y})$ is one-to-one, for the weighting scheme $\lambda=\left\{\lambda_{k}\right\}_{k=2}^{\infty}$ such that $\sum_{k=2}^{\infty} \lambda_{k}=1$.

Below, we give a sufficient condition for invertibility of $\mathcal{T}_{\lambda}$. Given $\left\{\omega_{l}\right\}_{l=1}^{L} \subset \mathcal{Y}$, we define a marginal discretization (with respect to $y_{t-k}$ ) of the joint density function $f_{\lambda}(\cdot, \cdot)$ by $\left[f_{\lambda}\left(x, \omega_{1}\right), \ldots, f_{\lambda}\left(x, \omega_{L}\right)\right]^{\top}$.

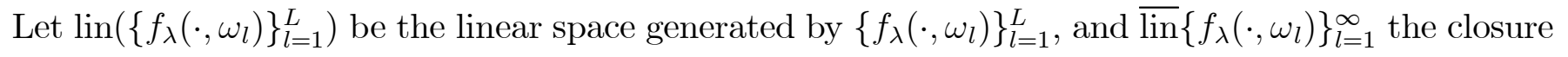
of $\operatorname{lin}\left\{f_{\lambda}\left(\cdot, \omega_{l}\right)\right\}_{l=1}^{\infty}$ in $L^{2}(\mathcal{Y})$. Our identification results make use of the following condition.

A.1. For the given sequence $\lambda=\left\{\lambda_{k}\right\}_{k=2}^{\infty}$, satisfying $\lambda_{k} \geq 0$ and $\sum_{k=2}^{\infty} \lambda_{k}=1$, for some sequence $\overline{\mathcal{Y}}=\left\{\omega_{l}\right\}_{l=1}^{\infty} \subset Y, \operatorname{lin}\left\{f_{\lambda}\left(\cdot, \omega_{l}\right)\right\}_{l=1}^{\infty}$ is dense in $L^{2}(Y)$, i.e., $\overline{\operatorname{lin}}\left\{f_{\lambda}\left(\cdot, \omega_{l}\right)\right\}_{l=1}^{\infty}=L^{2}(\mathcal{Y})$.

The above condition concerns richness of the linear spaces that are generated by a weighted sum of (unnormalized) conditional density function. A.1 will hold if a complete orthogonal basis of $L^{2}(\mathcal{Y})$ is generated by linear combinations of $\left\{f_{\lambda}\left(\cdot, \omega_{l}\right)\right\}_{l=1}^{\infty}$. The following theorem shows that A.1 is sufficient for $\mathcal{T}_{\lambda}$ to be one-to-one. The proof is immediate from Kim (2003, Theorem 2.2 (i), p.7).

Proposition 3.1 (i) If A.1 holds for some $k \geq 2$, then, the integral operator $\mathcal{T}_{\lambda}: L^{2}(\mathcal{Y}) \rightarrow$ $L^{2}(\mathcal{Y})$ is one-to-one, and $m_{0}(\cdot)$ is identified by $\mathcal{T}_{\lambda}^{-1}\left(h_{\lambda}\right) \in L^{2}(\mathcal{Y})$, for $h_{\lambda} \in \mathcal{R}\left(\mathcal{T}_{\lambda}\right)$.

The suggested identifying condition seems rather abstract, partly because we do not use any parametric assumptions. Roughly speaking, identifiability depends on the way that the density 
function of $y_{t-1}$, conditional on $y_{t-k}=\omega_{l}$, (or their weighted version) varies over different values of $\omega_{l}$ 's. For example, the model is identifiable, if some sequence of the conditional density functions, $\left\{f_{\mid \lambda}\left(\cdot \mid \omega_{l}\right)\right\}_{l=1}^{\infty}$, includes (or spans) a complete basis of $L^{2}(\mathcal{Y})$. A.1 excludes a joint density function of form $f_{k}(x, w)=\sum_{k=1}^{K} p_{k}(x) q_{k}(w)$ for finite $K$.

REMARK 1. Since $E\left(\eta_{t} \mid \mathcal{F}_{t-k}\right)=E\left(\eta_{t-1} \mid \mathcal{F}_{t-k}\right)=0$, for any $k \geq 2$, one may think of using a (non)linear function of $\left(y_{t-k}, y_{t-k-1}, \ldots,\right)$ as an instrument - a possibility that is not covered by the consideration above. Because of the curse of dimensionality arising from high dimensional conditioning variables, we will work only with moment conditions conditionalized on a single instrument. In this context, an alternative approach will be to use $w_{t}^{*}=\sum_{k \geq 2} \lambda_{k} y_{t-k}$ as an instrument. Note that, even when A.1 holds for no $\lambda$, a similar condition may hold for $w_{t}^{*}$.

Finally, we turn to the parametric term $\beta$. With $m_{0}$ given by Proposition 1 , the GARCH coefficient $\beta_{0}$ can be identified from the expected Gaussian likelihood function ${ }^{1}$

$$
l(\beta)=\mathrm{E}\left[\ln \sigma_{t}^{2}\left(\beta, m_{0}\right)+\frac{y_{t}^{2}}{\sigma_{t}^{2}\left(\beta, m_{0}\right)}\right],
$$

that is $\beta_{0}=\arg \min _{\beta \in B} l(\beta)$, where $B$ is a compact subset of $(0,1)$, while

$$
\sigma_{t}^{2}\left(\beta, m_{0}\right)=\sum_{j=1}^{T-1} \beta^{j-1}\left[(1-\beta) y_{t-j}^{2}+m\left(y_{t-j}\right)\right] .
$$

\section{Estimation}

We suppose that the quantities $h_{k}$ and $f_{k}$ are unknown but that there is an observed sample $\left\{y_{t}\right\}_{t=1}^{T}$. We shall assume now that the operator $\mathcal{T}_{\lambda}$ is invertible.

\subsection{Nonparametric Term}

Let $\tau$ be a truncation parameter satisfying $\tau(T) \rightarrow \infty$ as $T \rightarrow \infty$, and let $T^{*}=T-\tau$. We propose to estimate the quantities $h_{k}$ and $f_{k}$ from the sample data. Specifically, define

$$
\begin{gathered}
\widehat{h}_{k}(w)=\frac{1}{T^{*}} \sum_{t=\tau+1}^{T} K_{g_{2}}\left(y_{t-k}-w\right) \Delta y_{t}^{2}, \\
\widehat{f_{k}}(x, w)=\frac{1}{T^{*}} \sum_{t=\tau+1}^{T} K_{g_{1}}\left(y_{t-1}-x\right) K_{g_{2}}\left(y_{t-k}-w\right),
\end{gathered}
$$

\footnotetext{
${ }^{1}$ It should be noted that the least squares method is not consistent, since the second moment of $y_{t}$ does not exist.
} 


$$
\left(\widehat{\mathcal{T}}_{k} m\right)(w)=\int \frac{1}{T^{*}} \sum_{t=\tau+1}^{T} K_{g_{1}}\left(y_{t-1}-x\right) K_{g_{2}}\left(y_{t-k}-w\right) m(x) d x,
$$

where $K_{g}(s)=K(s / g) / g$, with $K(\cdot)$ being a symmetric function defined on the real line, while $g_{1}$ and $g_{2}$ are positive bandwidths.

We next solve the implied random integral equation to give our estimate of $m_{0}$. Let $\widehat{h}_{\lambda}(w)=$ $\sum_{k=2}^{\tau} \lambda_{k} \widehat{h}_{k}$ and $\widehat{\mathcal{T}}_{\lambda}=\sum_{k=2}^{\tau} \lambda_{k} \widehat{\mathcal{T}}_{k}$. Then consider the random Fredholm integral equation of the first kind,

$$
\left(\widehat{\mathcal{T}}_{\lambda} m\right)(w)=\int_{\mathcal{Y}} m(x) \widehat{f}_{\lambda}(x, w) d x=\widehat{h}_{\lambda}(w),
$$

where $\widehat{f}_{\lambda}(y, w)=\sum_{k=2}^{\tau} \lambda_{k} \widehat{f}_{k}(y, w)$. As is well known in mathematical inverse problems, several difficulties arise in estimating $m_{0}$ by inverting $\widehat{h}_{\lambda}$ through $\widehat{\mathcal{T}}_{\lambda}$.

Since $\widehat{\mathcal{T}}_{\lambda}$ is generally of finite rank, it is likely that $\widehat{h}_{\lambda} \notin \mathcal{R}\left(\widehat{\mathcal{T}}_{\lambda}\right)$, or $\widehat{\mathcal{T}}_{\lambda}$ is not invertible, i.e., the integral equation in (9) may possess no solution or more than one solutions. One resolve the existence and uniqueness problems easily by using the (Moore-Penrose) generalized inverse of $\widehat{\mathcal{T}}_{\lambda}$ :

$$
\widehat{m}^{\dagger}=\underset{m(\cdot) \in L^{2}(\mathcal{Y})}{\arg \min }\left\|\widehat{\mathcal{T}}_{\lambda} m-\widehat{h}_{\lambda}\right\|_{L^{2}(\mathcal{Y})}^{2}
$$

where $\widehat{m}^{\dagger}$ is the solution of minimum norm, unless the minimum-distance estimator is unique. Consistency of the natural estimator $\widehat{m}^{\dagger}$, however, is not ensured by consistency of the preliminary estimates $\widehat{h}_{\lambda}$ and $\widehat{\mathcal{T}}_{\lambda}$, since $\widehat{\mathcal{T}}_{\lambda}^{\dagger}$ is not bounded uniformly in $T$. Let $\|\mathcal{T}\|_{L^{2}(\mathcal{Y}) \rightarrow L^{2}(\mathcal{Y})}$ denote an operator norm of $\mathcal{T}: L^{2}(\mathcal{Y}) \rightarrow L^{2}(\mathcal{Y})$, given by $\sup _{m \in L^{2}(\mathcal{Y}), m \neq 0}\|\mathcal{T} m\|_{L^{2}(\mathcal{Y})} /\|m\|_{L^{2}(\mathcal{Y})}$. We say that $\widehat{\mathcal{T}}_{\lambda}: L^{2}(Y) \rightarrow L^{2}(Y)$ is uniformly consistent for $\mathcal{T}_{\lambda}$ on $M_{Y}$, if and only if $\left\|\widehat{\mathcal{T}}_{\lambda}-\mathcal{T}_{\lambda}\right\|_{L^{2}(\mathcal{Y}) \rightarrow L^{2}(\mathcal{Y})} \stackrel{p}{\rightarrow} 0$.

Proposition 4.1. Suppose that $\mathcal{T}_{\lambda}$ invertible. Assume that $\widehat{\mathcal{T}}_{\lambda}: L^{2}(\mathcal{Y}) \rightarrow L^{2}(\mathcal{Y})$ is uniformly consistent for $T_{\lambda}$ on $M_{Y} \subset L^{2}(Y)$ such that $\operatorname{dim}\left(M_{Y}\right)=\infty$. Then,

$$
\operatorname{plim}_{T \rightarrow \infty}\left\|\widehat{\mathcal{T}}_{\lambda}^{\dagger}\right\|_{L^{2}(\mathcal{Y}) \rightarrow L^{2}(\mathcal{Y})}=\infty .
$$

The naive estimator lacks stability with respect to the statistical errors in $\widehat{\mathcal{T}}_{\lambda}$ or $\widehat{h}_{\lambda}$. Small perturbations of $\widehat{\mathcal{T}}_{\lambda}$ or $\widehat{h}_{\lambda}$ may result in unacceptably large errors in $\widehat{m}^{\dagger}=\widehat{\mathcal{T}}_{\lambda}^{\dagger}\left(\widehat{h}_{\lambda}\right)$. Note that the estimation problem in (9) is statistically ill-posed, since the underlying mapping from $h_{\lambda}$ to $m$ is not continuous. For consistent estimation, some regularization is necessary.

\subsubsection{Tikhonov Regularization Method}

Define the adjoint operator

$$
\left(\widehat{\mathcal{T}}_{k}^{*} h\right)(y)=\int\left[\frac{1}{T^{*}} \sum_{t=\tau+1}^{T} K_{g_{1}}\left(y_{t-1}-y\right) K_{g_{2}}\left(y_{t-k}-w\right)\right] h(w) d w .
$$


By Fubini's Theorem,

$$
<\widehat{\mathcal{T}}_{\lambda} m, h>_{L^{2}(\mathcal{Y})}=<m, \widehat{\mathcal{T}}_{\lambda}^{*} h>_{L^{2}(\mathcal{Y})} \text { a.s. }
$$

and hence the two random operators $\widehat{\mathcal{T}}_{\lambda}$ and $\widehat{\mathcal{T}}_{\lambda}^{*}$, where $\widehat{\mathcal{T}}_{\lambda}^{*}=\sum_{k=2}^{\tau} \lambda_{k} \widehat{\mathcal{T}}_{k}^{*}$, are adjoint to each other. From $\operatorname{dim}\left(\mathcal{R}\left(\widehat{\mathcal{T}}_{\lambda}\right)\right) \leq T$, it follows that both $\widehat{\mathcal{T}}_{\lambda}$ and the self-adjoint operator $\widehat{\mathcal{T}}_{\lambda}^{*} \widehat{\mathcal{T}}_{\lambda}: L^{2}(\mathcal{Y}) \rightarrow L^{2}(\mathcal{Y})$ are bounded and compact.

We define a kernel IV estimator, based on the ordinary Tikhonov regularization, as

$$
\widehat{m}_{\alpha}=\left(\widehat{\mathcal{T}}_{\lambda}^{*} \widehat{\mathcal{T}}_{\lambda}+\alpha I\right)^{-1} \widehat{\mathcal{T}}_{\lambda}^{*} \widehat{h}_{\lambda}
$$

From the fact that $\widehat{\mathcal{T}}_{\lambda}^{*} \widehat{\mathcal{T}}_{\lambda}$ is self-adjoint, $\left(\widehat{\mathcal{T}}_{\lambda}^{*} \widehat{\mathcal{T}}_{\lambda}+\alpha I\right)^{-1}$ is well defined based on spectral theory for self-adjoint linear operators, since the real-valued function $U_{\alpha}(\kappa)=(\alpha+\kappa)^{-1}$ is well defined on the spectrum of $\widehat{\mathcal{T}}_{\lambda}^{*} \widehat{\mathcal{T}}_{\lambda}$.

To show the closed form of the kernel estimator, we need the following definitions. For

$$
\begin{gathered}
K_{T}^{Y}(x)=\left[K_{g_{1}}\left(y_{\tau}-x\right), \ldots, K_{g_{1}}\left(y_{T-1}-x\right)\right]^{\top}, \\
K_{T}^{\lambda}(w)=\left[\sum_{k=2}^{\tau} \lambda_{k} K_{g_{2}}\left(y_{\tau-k+1}-w\right), \ldots, \sum_{k=2}^{\tau} \lambda_{k} K_{g_{2}}\left(y_{T-k}-w\right)\right]^{\top},
\end{gathered}
$$

define

$$
M_{Y}=\int_{\mathcal{Y}} K_{T}^{Y}(x) K_{T}^{Y}(x)^{\top} d x \quad \text { and } \quad M_{\lambda}=\int_{\mathcal{Y}} K_{T}^{\lambda}(w) K_{T}^{\lambda}(w)^{\top} d w .
$$

Using a convolution-kernel function, we can rewrite the $(i, j)$-th element of $M_{\lambda}$, for example, in a more compact way, as

$$
\begin{aligned}
M_{i j}^{\lambda} & =\int_{\mathcal{Y}} \sum_{k=2}^{\tau} \lambda_{k} K_{g_{2}}\left(y_{\tau-k+i}-w\right) \sum_{l=2}^{\tau} \lambda_{l} K_{g_{2}}\left(y_{\tau-l+j}-w\right) d w \\
& =\sum_{k=2}^{\tau} \sum_{l=2}^{\tau} \lambda_{k} \lambda_{l} \int_{\mathcal{Y}} K_{g_{2}}\left(y_{\tau-k+i}-w\right) K_{g_{2}}\left(y_{\tau-l+j}-w\right) d w \\
& =\sum_{k=2}^{\tau} \sum_{l=2}^{\tau} \lambda_{k} \lambda_{l} K_{g_{2}}^{c}\left(y_{\tau-k+i}-y_{\tau-l+j}\right)
\end{aligned}
$$

where $K_{g_{2}}^{c}(w)=\left(1 / g_{2}\right) \int_{\mathcal{Y}} K\left(w / g_{2}-s\right) K(s) d s$. A straightforward calculation shows that $M_{\lambda}$ is a $\left(T^{*} \times T^{*}\right)$ symmetric nonnegative semi-definite matrix, for which the square-root matrix $M_{\lambda}^{1 / 2}$, satisfying $M_{\lambda}=M_{\lambda}^{1 / 2} M_{\lambda}^{1 / 2}$, is well-defined. ${ }^{2} \quad$ Also, define $Q_{Y, \lambda}=T^{*-2} M_{\lambda}^{1 / 2} M_{Y} M_{\lambda}^{1 / 2} ; Q_{Y, \lambda}$ is a $\left(T^{*} \times T^{*}\right)$ symmetric nonnegative semi-definite matrix, whose eigenvalues are all real and positive. We denote, by $\lambda_{\max }\left(Q_{Y, \lambda}\right)$, the maximum of those eigenvalues. We can show that

$$
{ }^{2} a^{\top} M_{\lambda} a=\sum_{1 \leq i, j \leq n} a_{i} M_{i j}^{\lambda} a_{j}=\int_{\mathcal{Y}}\left[\sum_{k} \lambda_{k} \sum_{i=1}^{n} a_{i} K_{g}\left(y_{\tau+i-k}-w\right)\right]^{2} d w \geq 0 \text {, for any } a(\neq 0) \in \mathbb{R}^{n} .
$$




$$
\widehat{m}_{\alpha}(x)=\left[\left(\alpha I+\widehat{\mathcal{T}}_{\lambda}^{*} \widehat{\mathcal{T}}_{\lambda}\right)^{-1} \widehat{\mathcal{T}}_{\lambda}^{*} \widehat{h}_{\lambda}\right](x)=T^{*-2} K_{T}^{Y}(x)^{\top} M_{\lambda}^{1 / 2}\left(Q_{Y, \lambda}+\alpha I_{T}\right)^{-1} M_{\lambda}^{1 / 2} \mathbf{y},
$$

where $\mathbf{y}=\left(\Delta y_{\tau+1}^{2}, \ldots, \Delta y_{T}^{2}\right)^{\top}$.

By (12), the abstract operator-form of the kernel estimator translates into a concrete matrixform. Computations of $\widehat{m}_{\alpha}$ only involve simple operations on finite-dimensional matrices, when the convolution-kernel weights in $M_{Y}$ and $M_{\lambda}$ are given.

Remark 4.1. (i) Suppose that $K(\cdot)$ is a density function from a stable distribution, say, a Gaussian kernel. Then, a further simplification of the convolution-kernel weight is available:

$$
M_{i j}^{\lambda}=\sum_{k=2}^{\tau} \sum_{l=2}^{\tau} \lambda_{k} \lambda_{l} K_{g_{2}}^{c}\left(y_{\tau-k+i}-y_{\tau-l+j}\right)=\sum_{k=2}^{\tau} \sum_{l=2}^{\tau} \lambda_{k} \lambda_{l} K_{\sqrt{2} g_{2}}\left(y_{\tau-k+i}-y_{\tau-l+j}\right),
$$

from $K^{c}(s)=K(s / \sqrt{2}) / \sqrt{2}$, since, by the stability assumption, the shape of a convoluted density function is not changed, except that the variance doubles. In that case, all the matrices in (12) are calculated in a straightforward way. In general, when there is no explicit form for the convolution kernel, we can compute $K^{c}(\cdot)$ by numerical integration.

(ii) By the spectral representation results in the proof of Theorem 4.2, the naive minimum-distance estimator in the above has a closed form

$$
\widehat{m}^{\dagger}(x)=K_{T}^{Y}(x)^{\top} M_{\lambda}^{1 / 2}\left(M_{\lambda}^{1 / 2} M_{Y} M_{\lambda}^{1 / 2}\right)^{\dagger} M_{\lambda}^{1 / 2} \mathbf{y} .
$$

If both $K_{T}^{Y}(\cdot)$ and $K_{T}^{\lambda}(\cdot)$ are assumed to be linearly independent, then, $M_{\lambda}$ and $M_{Y}$ are positive definite, from which we get $\widehat{m}^{\dagger}(x)=K_{T}^{Y}(x)^{\top} M_{Y}^{-1} \mathbf{y}$. From

$$
\begin{aligned}
\left(\widehat{\mathcal{T}}_{\lambda} \widehat{m}^{\dagger}\right)(w) & =\int \widehat{f}_{\lambda}(x, w) \widehat{m}^{\dagger}(x) d x=\frac{1}{T^{*}} K_{T}^{\lambda}(w)^{\top}<K_{T}^{Y}(\cdot), K_{T}^{Y^{\top}}(\cdot)>_{L^{2}(\mathcal{Y})} M_{Y}^{-1} \mathbf{y} \\
& =\frac{1}{T^{*}} K_{T}^{\lambda}(w)^{\top} \mathbf{y}=\widehat{h}_{\lambda}(w),
\end{aligned}
$$

we can confirm that $\widehat{m}^{\dagger}(\cdot)$ is one of the exact solutions to the integral equation, $\widehat{\mathcal{T}}_{\lambda} m=\widehat{h}_{\lambda}$, where $\widehat{\mathcal{T}}_{\lambda}$ will not be invertible in general. By the definition of the generalized inverse, $\widehat{m}^{\dagger}(\cdot)$ will be the solution of minimum-norm. Instability of $\widehat{m}^{\dagger}$ is obvious from the minimum eigenvalue of $M_{Y}$ converging to zero, as $T \rightarrow \infty$, since a pair of elements in $K_{T}^{Y}(\cdot)$ should become arbitrarily close to each other.

\subsection{Parametric term}

With a nonparametric estimate of $m_{0}$ given by $\widehat{m}_{\alpha}(\cdot)$ in the previous section, the parametric GARCH coefficient $\beta$ can be estimated by

$$
\widehat{\beta}=\arg \min _{\beta \in B} \widehat{\ell}(\beta)
$$


where $B$ is a compact subset of $(0,1)$ containing $\beta_{0}$, while

$$
\begin{aligned}
\widehat{\ell}(\beta) & =\frac{1}{T} \sum_{t=1}^{T} \ln \sigma_{t}^{2}\left(\beta, \widehat{m}_{\alpha}\right)+\frac{y_{t}^{2}}{\sigma_{t}^{2}\left(\beta, \widehat{m}_{\alpha}\right)} \\
\sigma_{t}^{2}\left(\beta, \widehat{m}_{\alpha}\right) & =\sum_{j=1}^{\min \left\{t-1, \tau_{\dagger}\right\}} \beta^{j-1}\left[(1-\beta) y_{t-j}^{2}+\widehat{m}_{\alpha}\left(y_{t-j}\right)\right] .
\end{aligned}
$$

Here, $\tau_{\dagger}=\tau_{\dagger}(T)<T$ is another truncation parameter. The estimator can be computed easily by a grid search over $B$.

\section{Asymptotic Properties}

\subsection{Nonparametric estimates}

Here we analyze the asymptotic properties of the kernel estimators proposed in the previous section. Let $\mathcal{F}_{b}^{a}$ be the $\sigma$-algebra of events generated by $\left\{y_{t}\right\}_{a}^{b}$ and $\alpha(k)$ the strong mixing coefficient of $\left\{y_{t}\right\}$ which is defined by

$$
\alpha(k) \equiv \sup _{A \in \mathcal{F}_{-\infty}^{0}, B \in \mathcal{F}_{k}^{\infty}}|P(A \cap B)-P(A) P(B)| .
$$

C.1 (a) $\left\{y_{t}\right\}_{t=1}^{\infty}$ is strictly stationary and strongly mixing (with a mixing coefficient, $\alpha(k)=\rho^{-\xi k}$, for some $\xi>0)$, and satisfies (1) with $m_{0}$ identified by $\mathcal{T}_{\lambda}^{-1} h_{\lambda}$. (b) $E\left(\mid \Delta y_{t}^{2} \| y_{t-k}=w\right)$ is bounded uniformly in $w$, a.s.

C.2 Let $K(\cdot) \in \mathbb{K}_{p^{*}}$, where $\mathbb{K}_{p^{*}}$ is the class of all Borel measurable symmetric real-valued functions $K(s)$ such that (a)

$$
\int|K(s)| d s<\infty, \quad \int K(s) d s=1, \quad \int K^{2}(s) d s<\infty, \quad \sup |K(s)|<\infty,
$$

and (b) $\int s^{j} K(s) d s=0$, for $j=1, \ldots, p^{*}-1$, and $\mu_{p^{*}}(K)=\int s^{p^{*}} K(s) d s<\infty$, where $p^{*}$ is an even integer.

C.3 The joint density functions $f_{k}(\cdot, \cdot)$ is square-integrable and bounded

$$
\sup _{k \geq 1} \int_{\mathcal{Y}} \int_{\mathcal{Y}} f_{k}^{2}(y, w) d y d w<\infty, \text { and } \sup _{k \geq 1} \sup _{(y, w) \in \mathcal{Y} \times \mathcal{Y}} f_{k}(y, w) \leq C<\infty .
$$

C.4 $f_{k}(\cdot, \cdot)$ and $m_{0}(\cdot)$ have continuous $p_{0}$-th and $p_{1}$-th partial derivatives, respectively, that are square-integrable, satisfying

$$
\sup _{k \geq 2}\left\|\frac{\partial^{p_{0}} f_{k}(y, w)}{\partial y^{q} \partial w^{p_{0}-q}}\right\|_{L^{2}(\mathcal{Y} \times \mathcal{Y})}^{2} \leq C, \text { and } \sup _{k \geq 2}\left\|\frac{d^{p_{1}} m(y)}{d y^{p_{1}}}\right\|_{L^{2}(\mathcal{Y})}^{2} \leq C
$$


C.5 (a) The bandwidth parameters $\left(g_{1}, g_{2}\right)$ satisfy that $\max \left(g_{1}, g_{2}\right) \rightarrow 0, T g_{2} \rightarrow \infty$. (b) The regularization parameter $\alpha$ satisfies that $\alpha \rightarrow 0, T g_{2} \alpha \rightarrow \infty$, and $g_{1}^{p_{0}} / \sqrt{\alpha} \rightarrow 0$, as $T \rightarrow \infty$.

C.6 $\left\{\lambda_{k}\right\}_{k=2}^{\infty}$ and $\tau=\tau_{T}$ are such that $\lambda_{k} \geq 0, \sum_{k=2}^{\infty} \lambda_{k}=1$, and $\sum_{k=\tau_{T}+1}^{\infty} \lambda_{k}=o(1 / \sqrt{T})$.

All the technical conditions in C.2 through C.4 are standard in nonparametric kernel estimation. As will be shown later, the $L^{2}$-convergence rate of our estimate can be derived under no requirement that the joint density functions have a compact support or be bounded away from zero. For uniform convergence results, however, the conditions in C.3 and C.4 will be strengthened, being replaced by a compact support assumption, together with the continuity condition. Note that the squareintegrability condition in C.3 entails boundedness of the linear operator $\mathcal{T}_{k}$. It can be satisfied under compact support with densities bounded away from zero, but it can also be satisfied in Gaussian process cases. See Linton and Mamen (2005) for further discussion. C.5(b), which is rather stronger than C.5(a), is necessary for consistency of the regularized kernel estimates. C.6 gives a convenient condition for controlling the approximation errors due to truncation. C.6 is satisfied, for example, when $\lambda_{k}=\lambda^{k}$ and $\tau_{T}=T^{-1 / m}$, for some positive (relatively large) integer. Let $\widehat{h}_{k}$ and $\widehat{\mathcal{T}}_{k}$ be given by (7) and (8), respectively. Our first result concerns sufficiency of the above conditions for derivation of the basic properties of the preliminary estimates, including consistency and the convergence rates.

Proposition 5.1 Suppose that C.1 through C.5(a) and C.6 hold. Then

$$
\begin{aligned}
\text { (i) }\left\|\widehat{\mathcal{T}}_{\lambda}-\mathcal{T}_{\lambda}\right\|_{L^{2}(\mathcal{Y}) \rightarrow L^{2}(\mathcal{Y})} & =O_{p}\left(1 / \sqrt{T g_{2}}+g_{1}^{p_{0}}+g_{2}^{p_{0}}\right), \\
\text { (ii) }\left\|\widehat{\mathcal{T}}_{\lambda}^{*}-\mathcal{T}_{\lambda}^{*}\right\|_{L^{2}(\mathcal{Y}) \rightarrow L^{2}(\mathcal{Y})} & =O_{p}\left(1 / \sqrt{T g_{1}}+g_{1}^{p_{0}}+g_{2}^{p_{0}}\right), \\
\text { (iii) }\left\|\widehat{h}_{\lambda}-\widehat{\mathcal{T}}_{\lambda} m_{0}\right\|_{L^{2}(\mathcal{Y})} & =O_{p}\left(1 / \sqrt{T g_{2}}+g_{1}^{\bar{p}}\right),
\end{aligned}
$$

where $\bar{p}=\max \left(p_{0}, p_{1}\right) \leq p^{*}$.

Noting that $U_{\alpha}(\kappa)=(\alpha+\kappa)^{-1}$ satisfies the conditions of C.3.1 and C.3.2 in $\operatorname{Kim}$ (2003, p.15), we can show the asymptotic properties of the kernel estimator $\widehat{m}_{\alpha}$, by applying the general results for statistical regularization (Kim, 2003, Theorem 3.3), together with Proposition 5.1. Let $\mathcal{N}(T)$ and $\mathcal{R}(T)$ denote the null space and range respectively of the operator $T$.

Theorem 5.2 Assume that C.1 through C.6 hold, with $p_{0}=p_{1}$. Then,

$$
\text { (i) }\left\|\widehat{m}_{\alpha}-m_{0}\right\|_{L^{2}(\mathcal{Y})} \stackrel{p}{\rightarrow} 0 \text {, as } T \rightarrow \infty \text {, for all } m_{0} \in L^{2}(\mathcal{Y}) \text {. }
$$

Assume additionally that $m_{0} \in \mathcal{R}\left(\mathcal{T}_{\lambda}^{*} \mathcal{T}_{\lambda}\right)$. Then,

$$
\text { (ii) }\left\|\widehat{m}_{\alpha}-m_{0}\right\|_{L^{2}(\mathcal{Y})} \leq O_{p}\left(\frac{1}{\sqrt{\alpha}}\left[\frac{1}{\sqrt{T g_{2}}}+g_{1}^{p_{0}}\right]\right)+O_{p}\left(\frac{1}{\sqrt{T g_{1}}}+g_{2}^{p_{0}}\right)+O_{p}(\alpha) \text {. }
$$


Since $\mathcal{T}_{\lambda}$ is an integral operator, the condition $m_{0} \in \mathcal{R}\left(\mathcal{T}_{\lambda}^{*} \mathcal{T}_{\lambda}\right)$ imposes certain smoothness on $m_{0}$, which we call an abstract smoothness condition. When $\mathcal{T}_{\lambda}$ is a compact operator, it means that the generalized Fourier coefficients of $m_{0}$ (with respect to the eigenfunctions) decay fast enough relative to the eigenvalues of $\mathcal{T}_{\lambda}{ }^{3}$

Remark 5.1. (the Optimal Convergence Rate) Let $m_{0}$ be any function in $\mathcal{R}\left(\mathcal{T}_{\lambda}^{*} \mathcal{T}_{\lambda}\right)$. Suppose a side condition on $\left(g_{1}, g_{2}\right)$ such that $\left(T g_{1}\right)^{-1 / 2} \leq O\left(g_{1}^{2 p_{0} / 3}\right)$, and $g_{2}^{3 p_{0} / 2} \leq O\left(\left[T g_{2}\right]^{-1 / 2}\right)$. Then, the optimal convergence rate of $\widehat{m}_{\alpha}$ is given by

$$
\left\|\widehat{m}_{\alpha}-m_{0}\right\|_{L^{2}(\mathcal{Y})}=O_{p}\left(T^{-\frac{p_{0}}{3 p_{0}+1}}\right),
$$

under the optimal choice of smoothing parameters such that $g_{1 T}^{*}=C_{0} T^{-\frac{1}{(4 / 3) p_{0}+1}}, g_{2 T}^{*}=C_{1} T^{-\frac{1}{3 p_{0}+1}}$, and $\alpha_{T}^{*}=C_{2} T^{-\frac{p_{0}}{3 p_{0}+1}}$.

Theorem 5.3 (uniform convergence rate of $\left.\widehat{m}_{\alpha}(x)\right)$ Assume that C.1 through C.6 hold $\left(p_{0}=p_{1}\right)$ with compactness of the support $\mathcal{Y}$, and that $m_{0} \in \mathcal{R}\left(\left[\mathcal{T}_{\lambda}^{*} \mathcal{T}_{\lambda}\right]^{\mu}\right)$, with $\mu \geq 1$. If $\alpha=o\left(1 /(\log T)^{c}\right)$, for any $c>0$, then,

$$
\sup _{y \in \mathcal{Y}}\left|\widehat{m}_{\alpha}(y)-m_{0}(y)\right|=O_{p}\left(\frac{\log T}{\sqrt{\alpha}}\left[\frac{1}{\sqrt{T g_{2}}}+g_{1}^{p_{0}}\right]\right)+O_{p}\left(\log T\left[\frac{1}{\sqrt{T g_{1}}}+g_{2}^{p_{0}}\right]\right)+O_{p}(\alpha \log T) .
$$

By using the same argument of Remark 5.1, we can show that the optimal uniform convergence rate of $\widehat{m}_{\alpha}$ is given by:

$$
\sup _{y \in \mathcal{Y}}\left|\widehat{m}_{\alpha}(y)-m_{0}(y)\right|=O_{p}\left(T^{-\frac{p_{0}}{3 p_{0}+1}} \log T\right), \quad \text { for } m_{0} \in \mathcal{R}\left(\mathcal{T}_{\lambda}^{*} \mathcal{T}_{\lambda}\right)
$$

where the optimal choice of smoothing parameters are the same as in Remark 5.1. Note that in this case, the twice-differentiability condition of $m$ and $f_{k}$ implies that

$$
\sup _{y \in \mathcal{Y}}\left|\widehat{m}_{\alpha}(y)-m_{0}(y)\right|=O_{p}\left(T^{-\frac{2}{7}} \log T\right)=o_{p}\left(T^{-1 / 4}\right) .
$$

\subsection{Parametric estimate}

We here establish the consistency with rate for the parametric part. With the uniform convergence rate of $\widehat{m}_{\alpha}(\cdot)$ faster than $T^{-1 / 4}$, the derivations are much simpler than those of Theorem 6 and the discussion in Section 4.4 in Linton and Mammen (2005), since $\widehat{m}_{\alpha}$ does not depend on $\widehat{\beta}$. First define the sample Gaussian quasi-likelihood (upto constants) assuming that $m_{0}$ is known perfectly,

$$
\ell(\beta)=\frac{1}{T} \sum_{t=1}^{T} \ln \sigma_{t}^{2}\left(\beta, m_{0}\right)+\frac{y_{t}^{2}}{\sigma_{t}^{2}\left(\beta, m_{0}\right)},
$$

\footnotetext{
${ }^{3}$ As in Tautenhahn (1998) and other papers in this literature we can use the weaker condition that $m_{0} \in$ $-\log \mathcal{R}\left(\left[\mathcal{T}_{\lambda}^{*} \mathcal{T}_{\lambda}\right]^{\eta}\right)$ for some $\eta>0$ at the cost of a great deal longer proofs.
} 
and suppose that $\widetilde{\beta}$ is the unique minimizer of $\ell(\beta)$, while $\beta_{0}$ is the unique minimizer of $E[\ell(\beta)]$. The properties of $\widetilde{\beta}$ have been well studied in the literature, see for example Lee and Hansen (1994) and Rahbek and Jensen (2004). In fact under the conditions that make $y_{t}$ strictly stationary and geometrically mixing we can expect $\widetilde{\beta}$ to be root- $\mathrm{T}$ consistent and asymptotically normal.

Theorem 5.4. Assume that C.1 through C.6 hold $\left(p_{0}=p_{1}\right)$ with compactness of the support $\mathcal{Y}$, and that $m_{0} \in \mathcal{R}\left(\left[\mathcal{T}_{\lambda}^{*} \mathcal{T}_{\lambda}\right]^{\mu}\right)$, with $\mu \geq 1$. Suppose that $\widetilde{\beta}$ is $T^{-1 / 4}$-consistent. If $\alpha=o\left(1 /(\log T)^{c}\right)$, for any $c>0$, and if $\tau_{\dagger}(T)=c \log T$, then,

$$
\widehat{\beta}-\beta=o_{p}\left(T^{-1 / 4}\right) \text {. }
$$

Under some conditions, one might be able to obtain asymptotic normality at rate root-T from the arguments of Chen and Shen (1998), but this is not guaranteed see Kim (2003).

\section{Numerical Results}

For an extensive simulation study of this method applied to a cross-sectional setting, see Kim (2003). We content ourselves with providing some evidence of the empirical relevance of our model and assumptions.

\subsection{Empirical Application}

The assumption that the process $\left\{\Delta y_{t}^{2}\right\}$ is weakly stationary while $y_{t}^{2}$ cannot be tested by examining the tail index of the two series. In practice one has to demean the process $y_{t}$ first. We investigate a sample of daily returns on the S\&P500 from 1955 to 2002, a total of 11,893 observations. The tail index $\kappa$ of a series $X_{t}$ is defined as the value for which

$$
1-\operatorname{Pr}\left(X_{t}>x\right) \simeq 1-L x^{-\kappa}
$$

as $x \rightarrow \infty$, where $L$ is a constant or a slowly varying function of $x$. We compute the tail index $\widehat{\kappa}$ of an ordered positive series $X_{t}$ by the Hill (1975) method

$$
\frac{1}{\widehat{\kappa}}=\frac{1}{M} \sum_{t=1}^{M} \log \frac{X_{t}}{X_{M+1}}
$$

In Figure 1 we give the Hill plot with $95 \%$ confidence interval for $y_{t}^{2}$. 
Figure 1. Shows the value of $\widehat{\kappa}$ against threshold size $M$ for the series $y_{t}^{2}$.

It is generally above one but less than two implying that $E\left[y_{t}^{2}\right]<\infty$ but $E\left[y_{t}^{4}\right]=\infty$. The corresponding hill plots for $\left|\Delta y_{t}^{2}\right|$ show slightly lighter tails: we just show the ratio of $\widehat{\kappa}$ for $\left|\Delta y_{t}^{2}\right|$ to $\widehat{\kappa}$ for $y_{t}^{2}$, which is generally above one. 
Figure 2. Shows the ratio of $\widehat{\kappa}$ for the series $\left|\Delta y_{t}^{2}\right|$ to $\widehat{\kappa}$ for the series $y_{t}^{2}$ against threshold size $M$.

For these data at least the evidence of integrated process is weak although it does seem that differencing reduces the weight of the tails significantly. Our second application is to a high frequency stock return series with $n=4626$. 
Figure 3. Shows the value of $\widehat{\kappa}$ against threshold size $M$ for the series $y_{t}^{2}$. High frequency stock return series. 
Figure 4. Shows the ratio of $\widehat{\kappa}$ for the series $\left|\Delta y_{t}^{2}\right|$ to $\widehat{\kappa}$ for the series $y_{t}^{2}$ against threshold size $M$. High frequency stock return series.

In this case, the differencing operation reduces the thickness of the tails considerably. The squared return series shows some evidence of non existence of first moment, but the differenced series has much lighter tails.

\section{Conclusions}

We established the consistency and rate of convergence of our estimates. Unfortunately, the pointwise distribution theory for $\widehat{m}$ appears to be very difficult and we have not anything to offer on this. Likewise our theory for $\widehat{\beta}$ falls short of root-T consistency and asymptotic normality. 


\section{Appendix}

Proof of Proposition 4.1 The integral operator $\widehat{\mathcal{T}}_{\lambda}$ has a degenerate kernel, i.e., $\widehat{f}_{\lambda}(\cdot, \cdot)$ is a finite sum of products of kernel weights on each observation. Noting that $\widehat{\mathcal{T}}_{\lambda}$ is of a finite rank, the proof is direct from Proposition 3.1 in $\operatorname{Kim}(2003)$, since $\widehat{\mathcal{T}}_{\lambda}: L^{2}(\mathcal{Y}) \rightarrow L^{2}(\mathcal{Y})$ is uniformly consistent for $\mathcal{T}_{\lambda}$ on $\mathcal{M}_{Y} \subset L^{2}(\mathcal{Y})$ s.t. $\operatorname{dim}\left(\mathcal{M}_{Y}\right)=\infty$.

Lemma A.1. Assume that C.1 through C.5(a) hold. Then, it holds that

$$
\begin{array}{r}
(i) \sup _{k \geq 2}\left\|\widehat{\mathcal{T}}_{k}-\mathcal{T}_{k}\right\|^{2}=O_{p}\left(g_{1}^{2 p_{0}}+g_{2}^{2 p_{0}}\right)+O_{p}\left(\frac{1}{T g_{2}}\right), \\
\left(\text { ii) } \sup _{k \geq 2}\left\|\widehat{\mathcal{T}}_{k}^{*}-\mathcal{T}_{k}^{*}\right\|^{2}=O_{p}\left(g_{1}^{2 p_{0}}+g_{2}^{2 p_{0}}\right)+O_{p}\left(\frac{1}{T g_{1}}\right),\right. \\
(\text { iii }) \sup _{k \geq 2}\left\|\widehat{h}_{k}-\widehat{\mathcal{T}}_{k} m_{0}\right\|_{L^{2}(\mathcal{Y})}^{2}=O_{p}\left(\frac{1}{T g_{2}}\right)+O_{p}\left(g_{1}^{2 \bar{p}}+g_{2}^{2 p_{0}}\right),
\end{array}
$$

where $\bar{p}=\max \left(p_{0}, p_{1}\right)$.

Proof. (i) With $a * b$ denoting convolution of $a$ and $b$, we define

$$
m_{c\left(g_{1}\right)}(y) \equiv\left(K_{g_{1}} * m\right)(y)=\int K_{g_{1}}(y-s) m(s) d s,
$$

and

$$
\begin{aligned}
f_{k}^{c(g)}(y, w) & \equiv\left(K_{\left(g_{1}, g_{2}\right)} * f_{k}\right)(y, w)=\int_{\mathcal{Y}} \int_{\mathcal{Y}} K_{g_{1}}\left(s_{1}-y\right) K_{g_{2}}\left(s_{2}-w\right) f_{k}\left(s_{1}, s_{2}\right) d s_{1} d s_{2} \\
& =\mathrm{E}\left[K_{g_{1}}\left(y_{t-1}-y\right) K_{g_{2}}\left(y_{t-k}-w\right)\right] .
\end{aligned}
$$

By adding and subtracting $\int f_{k}^{c(g)}(y, w) m(y) d y$, the estimation errors of $\widehat{\mathcal{T}}_{k}$ are decomposed into

$$
\begin{aligned}
\left(\widehat{\mathcal{T}}_{k} m-\mathcal{T}_{k} m\right)(w)= & \frac{1}{T^{*}} \sum_{t=\tau+1}^{T} \int\left[K_{g_{1}}\left(y_{t-1}-y\right) K_{g_{2}}\left(y_{t-k}-w\right)-f_{k}^{c(g)}(y, w)\right] m(y) d y \\
& +\int\left[f_{k}^{c(g)}(y, w)-f_{k}(y, w)\right] m(y) d y \\
\equiv & \mathrm{s}_{T}(w)+\mathrm{B}_{T}(w),
\end{aligned}
$$

from which we obtain the MISE of $\widehat{\mathcal{T}}_{k} m$, given by

$$
\mathrm{E} \int_{\mathcal{Y}}\left[\left(\widehat{\mathcal{T}}_{k}-\mathcal{T}_{k}\right) m\right]^{2}(w) d w=\int_{\mathcal{Y}}\left\{\operatorname{Var}\left[\mathrm{s}_{T}(w)\right]+\mathrm{E}^{2}\left[\mathrm{~B}_{T}(w)\right]\right\} d w .
$$

From

$$
\mathrm{s}_{T}(w)=\frac{1}{T^{*}} \sum_{t=\tau+1}^{T}\left\{K_{g_{2}}\left(y_{t-k}-w\right) m_{c\left(g_{1}\right)}\left(y_{t-1}\right)-\mathrm{E}\left[K_{g_{2}}\left(y_{t-k}-w\right) m_{c\left(g_{1}\right)}\left(y_{t-1}\right)\right]\right\}
$$


a standard calculation of the variance term for kernel estimates (under the mixing condition in C.1) yields

$$
\begin{aligned}
\operatorname{Var}\left[s_{T}(w)\right] & =\frac{1}{T^{*}} \operatorname{Var}\left[K_{g_{2}}\left(y_{t-k}-w\right) m_{c\left(g_{1}\right)}\left(y_{t-1}\right)\right]+o\left(\frac{1}{T g_{2}}\right) \\
& =\frac{1}{T g_{2}}\|K\|_{2}^{2} \int_{\mathcal{Y}} m_{c\left(g_{1}\right)}^{2}(y) f_{k}(y, w) d y+O\left(T^{-1}\right)+o\left(\frac{1}{T g_{2}}\right),
\end{aligned}
$$

where the last equality is due to the dominated convergence theorem. Let $f_{0}(\cdot)$ be the marginal density function of $y_{t}$. Since $f_{0}(\cdot)$ is assumed to be uniformly bounded (condition C.3), it holds

$$
\begin{aligned}
\int_{\mathcal{Y}} \operatorname{Var}\left[s_{T}(w)\right] d w & \simeq \frac{1}{T g_{2}}\|K\|_{2}^{2} \int_{\mathcal{Y}} m^{2}(y) f_{0}(y) d y \\
& \leq C \frac{1}{T g_{2}}\|K\|_{2}^{2}\|m\|_{L^{2}(\mathcal{Y})}^{2} .
\end{aligned}
$$

To calculate the bias term, we observe that, by Cauchy-Schwarz inequality,

$$
\begin{aligned}
\int_{\mathcal{Y}} \mathrm{B}_{T}^{2}(w) d w & =\int_{\mathcal{Y}}\left[\int_{\mathcal{Y}}\left\{f_{k}^{c}(y, w)-f_{k}(y, w)\right\} m(y) d y\right]^{2} d w \\
& \leq\left\|f_{k}^{c}-f_{k}\right\|_{L^{2}(\mathcal{Y} \times \mathcal{Y})}^{2}\|m\|_{L^{2}(\mathcal{Y})}^{2}
\end{aligned}
$$

Under C.2 (i.e., $\int|K(s)| d s<\infty$ and $\left.\sup |K(s)|<\infty\right)$, the convolution errors $\left(\left\|f_{k}^{c}-f_{k}\right\|_{L^{2}(\mathcal{Y} \times \mathcal{Y})}^{2}\right)$ converge to zero, as $g_{1}$ and $g_{2}$ go to zero, for any square integrable $f_{k}(\cdot, \cdot)$. When there exist $p_{0}$-th partial derivatives of $f_{k}(\cdot, \cdot)$ that are continuous and square integrable (i.e., condition C.4 holds), we have, by application of the standard Taylor expansion, that

$$
\int_{\mathcal{Y}} \mathrm{B}_{T}^{2}(w) d w \leq C\left\{g_{1}^{2 q} g_{2}^{2\left(p_{0}-q\right)} \sum_{q=0}^{p_{0}}\left\|\frac{\partial^{p_{0}} f_{k}(y, w)}{\partial y^{q} \partial w^{p_{0}-q}}\right\|_{L^{2}(\mathcal{Y} \times \mathcal{Y})}^{2}\right\}\|m\|_{L^{2}(\mathcal{Y})}^{2} .
$$

Combining (15) and (16), we obtain

$$
\mathrm{E} \int_{\mathcal{Y}}\left[\left(\widehat{\mathcal{T}}_{k} m-\mathcal{T}_{k} m\right)(w)\right]^{2} d w \leq C\|m\|_{L^{2}(\mathcal{Y})}^{2}\left(g_{1}^{2 q} g_{2}^{2\left(p_{0}-q\right)} \sum_{q=0}^{p_{0}}\left\|\frac{\partial^{p_{0}} f_{k}(y, w)}{\partial y^{q} \partial w^{p_{0}-q}}\right\|_{L^{2}(\mathcal{Y} \times \mathcal{Y})}^{2}+\frac{1}{T g_{2}}\|K\|_{2}^{2}\right),
$$

implying

$$
\begin{aligned}
\mathrm{E}\left\|\widehat{\mathcal{T}}_{k}-\mathcal{T}_{k}\right\|_{L^{2}(\mathcal{Y}) \rightarrow L^{2}(\mathcal{Y})}^{2} & =\sup _{m(\neq 0) \in L^{2}(\mathcal{Y})} \frac{\mathrm{E} \int_{\mathcal{Y}}\left[\left(\widehat{\mathcal{T}}_{k}-\mathcal{T}_{k}\right) m\right]^{2}(w) d w}{\|m\|_{L^{2}(\mathcal{Y})}^{2}} \\
& \leq C\left(\sum_{q=0}^{p_{0}} g_{1}^{2 q} g_{2}^{2\left(p_{0}-q\right)}\left\|\mid \frac{\partial^{p_{0}} f_{k}(y, w)}{\partial y^{q} \partial w^{p_{0}-q}}\right\|_{L^{2}(\mathcal{Y} \times \mathcal{Y})}^{2}+\frac{1}{T g_{2}}\|K\|_{2}^{2}\right) .
\end{aligned}
$$

Noting that the variance term in (15) does not depend on $k$ and $\sup _{k \geq 2}\left\|\frac{\partial^{p_{0}} f_{k}(y, w)}{\partial y^{q} \partial w^{p} p^{-q}}\right\|_{L^{2}(\mathcal{Y} \times \mathcal{Y})}^{2} \leq C$ (condition C.4), we finally get

$$
\sup _{k \geq 2} \mathrm{E}\left\|\left(\widehat{\mathcal{T}}_{k}-\mathcal{T}_{k}\right) m\right\|_{L^{2}(\mathcal{Y}) \rightarrow L^{2}(\mathcal{Y})}^{2}=O\left(g_{1}^{2 p_{0}}+g_{2}^{2 p_{0}}\right)+O\left(\frac{1}{T g_{2}}\right) .
$$


(ii) By symmetry of the above arguments, a similar result holds for $\widehat{\mathcal{T}}_{k}^{*}$.

(iii) Let $r\left(y_{t-1}\right)=m_{0}\left(y_{t-1}\right)-m_{0, c\left(g_{1}\right)}\left(y_{t-1}\right)$, where $m_{0, c\left(g_{1}\right)}(\cdot)$ is defined by $(14)$. From $\left(\widehat{\mathcal{T}}_{k} m\right)(w)=$ $T^{*-1} \sum_{t=\tau+1}^{T} K_{g_{2}}\left(y_{t-k}-w\right) m_{c\left(g_{1}\right)}\left(y_{t-1}\right)$, we get

$$
\begin{aligned}
& \left(\widehat{h}_{k}-\widehat{\mathcal{T}}_{k} m_{0}\right)(w) \\
= & \frac{1}{T^{*}} \sum_{t=\tau+1}^{T} K_{g_{2}}\left(y_{t-k}-w\right) \eta_{t}+\frac{1}{T^{*}} \sum_{t=\tau+1}^{T} K_{g_{2}}\left(y_{t-k}-w\right) r\left(y_{t-1}\right) \\
= & \frac{1}{T^{*}} \sum_{t=\tau+1}^{T} K_{g_{2}}\left(y_{t-k}-w\right) \eta_{t}+\frac{1}{T^{*}} \sum_{t=\tau+1}^{T} K_{g_{2}}\left(y_{t-k}-w\right)\left(\nu_{t-1}-\nu_{t-1}^{c}\right) \\
& +\frac{1}{T^{*}} \sum_{t=\tau+1}^{T} K_{g_{2}}\left(y_{t-k}-w\right) \mathrm{E}\left(r\left(y_{t-1}\right) \mid y_{t-k}\right) \\
\equiv & s_{1, T}(w)+s_{2, T}(w)+\mathrm{B}_{T}(w),
\end{aligned}
$$

where $\nu_{t-1}=m_{0}\left(y_{t-1}\right)-\mathrm{E}\left(m_{0}\left(y_{t-1}\right) \mid y_{t-k}\right)$, and $\nu_{t-1}^{c}=m_{0, c\left(g_{1}\right)}\left(y_{t-1}\right)-\mathrm{E}\left(m_{0, c\left(g_{1}\right)}\left(y_{t-1}\right) \mid y_{t-k}\right)$.

As a consequence,

$$
\mathrm{E} \int_{\mathcal{Y}}\left(\widehat{h}_{k}-\widehat{\mathcal{T}}_{k} m_{0}\right)^{2}(w) d w=\int_{\mathcal{Y}}\left\{\operatorname{Var}\left[s_{1, T}(w)+s_{2, T}(w)\right]+\mathrm{E}^{2}\left[\mathrm{~B}_{T}(w)\right]\right\} d w
$$

By the standard argument in kernel regression, the variance of the main stochastic term is calculated in a straightforward way:

$$
\operatorname{Var}\left[s_{1, T}(w)\right]=\frac{1}{T g_{2}}\|K\|_{2}^{2} \mathrm{E}\left(\eta_{t}^{2} \mid y_{t-k}=w\right) f_{0}(w)(1+o(1)),
$$

leading to

$$
\begin{aligned}
\int_{\mathcal{Y}} \operatorname{Var}\left[s_{1, T}(w)\right] d w & =\frac{1}{T g_{2}}\|K\|_{2}^{2}\left[\int_{\mathcal{Y}} \mathrm{E}\left(\eta_{t}^{2} \mid y_{t-k}=w\right) f_{0}(w) d w\right](1+o(1)) \\
& =\frac{1}{T g_{2}}\|K\|_{2}^{2} \sigma_{\eta}^{2}(1+o(1))=O\left(\frac{1}{T g_{2}}\right),
\end{aligned}
$$

where $\sigma_{\eta}^{2}=\mathrm{E}\left(\eta_{t}^{2}\right)$. From $\mathrm{E}\left[\nu_{t-1} \mid y_{t-k}\right]=\mathrm{E}\left[\nu_{t-1}^{c} \mid y_{t-k}\right]=0$, it follows that

$$
\mathrm{E}\left[\left(\nu_{t-1}-\nu_{t-1}^{c}\right)^{2} \mid y_{t-k}\right]=\operatorname{Var}\left[r\left(y_{t-1}\right) \mid y_{t-k}\right] \leq \mathrm{E}\left[r^{2}\left(y_{t-1}\right) \mid y_{t-k}\right],
$$

implying, by the mixing assumption and the law of iterated expectation, that

$$
\begin{aligned}
\operatorname{Var}\left[s_{2, T}(w)\right] & \simeq \frac{1}{T^{*}} \mathrm{E}\left\{\left[K_{g_{2}}\left(y_{t-k}-w\right)\left(\nu_{t-1}-\nu_{t-1}^{c}\right)\right]^{2}\right\} \\
& =\frac{1}{T^{*}} \mathrm{E}\left\{\left[K_{g_{2}}\left(y_{t-k}-w\right)\right]^{2} \mathrm{E}\left[r^{2}\left(y_{t-1}\right) \mid y_{t-k}\right]\right\} \\
& =\frac{1}{T^{*}} \mathrm{E}\left\{\left[K_{g_{2}}\left(y_{t-k}-w\right) r\left(y_{t-1}\right)\right]^{2}\right\} \\
& =\frac{1}{T g_{2}}\|K\|_{2}^{2} \int_{\mathcal{Y}} r^{2}(y) f_{k}(y, w) d y(1+o(1)) .
\end{aligned}
$$


From boundedness of $f_{0}(\cdot)$,

$$
\begin{aligned}
\int_{\mathcal{Y}} \operatorname{Var}\left[s_{2, T}(w)\right] d w & \simeq \frac{1}{T g_{2}}\|K\|_{2}^{2} \int_{\mathcal{Y}} r^{2}(y) f_{0}(y) d y \\
& \leq \frac{C}{T g_{2}}\|K\|_{2}^{2}\left\|m(\cdot)-m_{c\left(g_{1}\right)}(\cdot)\right\|_{L^{2}(\mathcal{Y})}^{2}=o\left(\frac{1}{T g_{2}}\right)
\end{aligned}
$$

since the convolution error, $\left\|m(\cdot)-m_{c\left(g_{1}\right)}(\cdot)\right\|_{L^{2}(\mathcal{Y})}$, converges to zero, as $g_{1} \rightarrow 0$. To calculate the bias term, we note, by the dominated convergence theorem, that

$$
\begin{aligned}
\mathrm{E}\left[\mathrm{B}_{T}(w)\right] & =\mathrm{E}\left[K_{g_{2}}\left(y_{t-k}-w\right) r\left(y_{t-1}\right)\right] \\
& =\int_{\mathcal{Y}}\left[m(y)-m_{c\left(g_{1}\right)}(y)\right] f_{k}(y, w) d y(1+o(1)) .
\end{aligned}
$$

Letting $f_{k}^{c\left(g_{1}\right)}(u, w)=\int_{\mathcal{Y}} K_{g_{1}}(y-u) f_{k}(y, w) d y$, we obtain an alternative form of the bias such that

$$
\mathrm{E}\left[\mathrm{B}_{T}(w)\right]=\left\{\int_{\mathcal{Y}}\left[f_{k}(u, w)-f_{k}^{c\left(g_{1}\right)}(u, w)\right] m(u) d u\right\}(1+o(1)),
$$

since $\int_{\mathcal{Y}} m_{c\left(g_{1}\right)}(z) f_{k}(z, w) d z=\int_{\mathcal{Y}} f_{k}^{c\left(g_{1}\right)}(u, w) m(u) d u$, by Fubini's Theorem. By Cauchy-Schwarz inequality, it follows from (18) and (19), together with square-integrability of $f_{k}(\cdot, \cdot)$ and $m(\cdot)$, that

$$
\int_{\mathcal{Y}} \mathrm{E}^{2}\left[\mathrm{~B}_{T}(w)\right] d w \leq C \min \left\{\left\|m(\cdot)-m_{c\left(g_{1}\right)}(\cdot)\right\|^{2},\left\|f_{k}(\cdot)-f_{k}^{c\left(g_{1}\right)}(\cdot)\right\|^{2}\right\}
$$

which, by the standard method of Taylor expansion (under C.4 ), gives

$$
\int_{\mathcal{Y}} \mathrm{E}^{2}\left[\mathrm{~B}_{T}(w)\right] d w=O\left(g_{1}^{\max \left\{2 p_{0}, 2 p_{1}\right\}}+g_{2}^{2 p_{0}}\right)
$$

Since the variance term in (17) dose not depend on $k$ and $\sup _{k \geq 2}\left\|\frac{d^{p_{1} m(y)}}{d y^{p_{1}}}\right\|_{L^{2}(\mathcal{Y})}^{2} \leq C$ (condition C.4), it holds that

$$
\sup _{k \geq 2} \mathrm{E} \int_{\mathcal{Y}}\left(\widehat{h}_{k}-\widehat{\mathcal{T}}_{k} m_{0}\right)^{2}(w) d w=O\left(\frac{1}{T g_{2}}\right)+O\left(g_{1}^{2 \bar{p}}+g_{2}^{2 p_{0}}\right)
$$

Proof of Proposition 5.1. (i) By the triangle inequality,

$$
\begin{aligned}
\left\|\widehat{\mathcal{T}}_{\lambda}-\mathcal{T}_{\lambda}\right\|_{L^{2}(\mathcal{Y}) \rightarrow L^{2}(\mathcal{Y})} & \leq \sum_{k=2}^{\tau} \lambda_{k}\left\|\widehat{\mathcal{T}}_{k}-\mathcal{T}_{k}\right\|_{L^{2}(\mathcal{Y}) \rightarrow L^{2}(\mathcal{Y})}+\sum_{k=\tau+1}^{\infty} \lambda_{k}\left\|\mathcal{T}_{k}\right\|_{L^{2}(\mathcal{Y}) \rightarrow L^{2}(\mathcal{Y})} \\
& \leq C\left\{\sup _{k \geq 2}\left\|\widehat{\mathcal{T}}_{k}-\mathcal{T}_{k}\right\|_{L^{2}(\mathcal{Y}) \rightarrow L^{2}(\mathcal{Y})}+o(1 / \sqrt{T})\right\}
\end{aligned}
$$

where the second inequality follows from $\sup _{k \geq 2}\left\|\mathcal{T}_{k}\right\| \leq \sup _{k \geq 2}\left\|f_{k}\right\| \leq C$ (condition C.3) and $\sum_{k=\tau+1}^{\infty} \lambda_{k}=o(1 / \sqrt{T})$ (condition C.6). Now, the proof is immediate from Lemma A.1(i). 
The proof of (ii) can be shown in the same way.

(iii) Note that

$$
\left\|\widehat{h}_{\lambda}-\widehat{\mathcal{T}}_{\lambda} m_{0}\right\| \leq \sum_{k=2}^{\tau} \lambda_{k}\left\|\widehat{h}_{k}-\widehat{\mathcal{T}}_{k} m_{0}\right\| \leq \sup _{k \geq 2}\left\|\widehat{h}_{k}-\widehat{\mathcal{T}}_{k} m_{0}\right\|
$$

since $\sum_{k=2}^{\tau} \lambda_{k} \leq \sum_{k=2}^{\infty} \lambda_{k}=1$. The proof follows, if we apply Lemma A.1(iii).

Before proving the main results, we need to introduce some useful lemmas that are borrowed from $\operatorname{Kim}(2003)$. First, note that $U_{\alpha}(\kappa)=(\alpha+\kappa)^{-1}$ satisfies the following conditions:

B.1 Let $\bar{\kappa} \equiv \sup _{n \geq n_{0}}\left\|\widehat{\mathcal{T}}_{\lambda}^{*} \widehat{\mathcal{T}}_{\lambda}\right\|_{L^{2}(\mathcal{Y}) \rightarrow L^{2}(\mathcal{Y})}$. A parameter dependent family of continuous functions, $\left\{U_{\alpha}(\cdot)\right\}_{\alpha>0}$, defined on $(0, \bar{\kappa}]$, satisfy that (i) $\sup _{\kappa \in(0, \bar{\kappa}]}\left|U_{\alpha}(\kappa) \kappa\right| \leq C<\infty$, for $\alpha>0$, (ii) $\lim _{\alpha \rightarrow 0^{+}} U_{\alpha}(\kappa)=\frac{1}{\kappa}$, for all $\kappa \in(0, \bar{\kappa}]$, and (iii) $\sup _{\kappa \in(0, \bar{\kappa}]}\left|U_{\alpha}(\kappa)\right|=O\left(\frac{1}{\alpha}\right)$, as $\alpha \rightarrow 0^{+}$.

B.2 Given $U_{\alpha}:(0, \bar{\kappa}] \rightarrow \mathbb{R}$, it holds for any $\mu \in(0,1]$ that $\sup _{\kappa \in(0, \bar{\kappa}]} \kappa^{\mu}\left|U_{\alpha}(\kappa) \kappa-1\right| \leq C \alpha^{\mu}$, for any $\alpha \in\left(0, \alpha_{0}\right)$, where $\alpha_{0}>0$.

Lemma A.2 If $\alpha=\alpha(T) \rightarrow 0$ as $T \rightarrow \infty$, then:

(i) $\quad\left\|U_{\alpha}\left(\widehat{\mathcal{T}}_{\lambda}^{*} \widehat{\mathcal{T}}_{\lambda}\right)\right\|_{L^{2}(\mathcal{Y}) \rightarrow L^{2}(\mathcal{Y})}=O_{a . s}\left(\alpha^{-1}\right)$,

(ii) $\quad\left\|U_{\alpha}\left(\widehat{\mathcal{T}}_{\lambda}^{*} \widehat{\mathcal{T}}_{\lambda}\right) \widehat{\mathcal{T}}_{\lambda}^{*}\right\|_{L^{2}(\mathcal{Y}) \rightarrow L^{2}(\mathcal{Y})}=O_{a . s}\left(\sqrt{\alpha}^{-1}\right)$,

Assume additionally that $\widehat{\mathcal{T}}_{\lambda}: L^{2}(\mathcal{Y}) \rightarrow L^{2}(\mathcal{Y})$ converges pointwise, in probability, to $\mathcal{T}_{\lambda}: L^{2}(\mathcal{Y}) \rightarrow$ $L^{2}(\mathcal{Y})$, which is bounded and one-to-one. Then,

(iii) $\left\|\left[U_{\alpha}\left(\widehat{\mathcal{T}}_{\lambda}^{*} \widehat{\mathcal{T}}_{\lambda}\right) \widehat{\mathcal{T}}_{\lambda}^{*} \widehat{\mathcal{T}}_{\lambda}-I\right] m\right\|_{L^{2}(\mathcal{Y})}=o_{p}(1)$, for all $m \in L^{2}(\mathcal{Y})$

Proof Since $U_{\alpha}(\cdot)$ satisfies B.1 and $\widehat{\mathcal{T}}_{\lambda}$ has a finite rank, the results are immediate from Lemma $3.2(\operatorname{Kim} 2003$, p.41).

Lemma A.3 Let $G: L^{2}(\mathcal{Y}) \rightarrow L^{2}(\mathcal{Y})$ be a linear bounded operator and $G^{*}: L^{2}(\mathcal{Y}) \rightarrow L^{2}(\mathcal{Y})$ be adjoint to $G$. Then, for all $m \in L^{2}(\mathcal{Y})$,

(i) $\quad\left\|\left[U_{\alpha}\left(G^{*} G\right) G^{*} G-I\right]\left(G^{*} G\right)^{\mu}\right\|_{L^{2}(\mathcal{Y}) \rightarrow L^{2}(\mathcal{Y})} \leq C \alpha^{\min (\mu, 1)}$, for $\mu>0$.

(ii) $\quad\left\|\left[U_{\alpha}\left(G^{*} G\right) G^{*} G-I\right] G^{*}\right\|_{L^{2}(\mathcal{Y}) \rightarrow L^{2}(\mathcal{Y})} \leq C \alpha^{1 / 2}$, for $\mu>0$.

Proof. Since $U_{\alpha}$ satisfies B.2, the results follow from Lemma 3.3 (Kim 2003, p.42). See also Carrasco, Florens and Renault (2006). 
For $\mathcal{M}_{T}(\mathcal{Y})=\left\{m \in \mathcal{M}(\mathcal{Y}): m_{T}(x)=T^{*-2} K_{T}^{Y}(x)^{\top} b_{T}\right.$, for $\left.b_{T} \in \mathbb{R}^{T^{*}}\right\}$, we define $\left(\widehat{\mathcal{T}}_{\lambda}^{*} \widehat{\mathcal{T}}_{\lambda}\right)_{\mid \mathcal{M}_{T}}^{\omega}:$ $\mathcal{M}_{T} \rightarrow C(\mathcal{Y})$ to be the restriction (into $\left.\mathcal{M}_{T}\right)$ of $\left(\widehat{\mathcal{T}}_{\lambda}^{*} \widehat{\mathcal{T}}_{\lambda}\right)^{\omega}: L^{2}(\mathcal{Y}) \rightarrow C(\mathcal{Y})$, where $C(\mathcal{Y})$ is a space of continuous functions defined on $\mathcal{Y}$.

Lemma A.4. Assume that $\widehat{f}_{k}(\cdot, \cdot)$ is a uniformly consistent estimate of $f_{k}(\cdot, \cdot)$ which is continuous with a compact support. Then, $\left(\widehat{\mathcal{T}}_{\lambda}^{*} \widehat{\mathcal{T}}_{\lambda}\right)_{\mid \mathcal{M}_{T}}^{\omega}: \mathcal{M}_{T} \rightarrow C(\mathcal{Y})$ is uniformly bounded in the sense that

$$
\left\|\left(\widehat{\mathcal{T}}_{\lambda}^{*} \widehat{\mathcal{T}}_{\lambda}\right)_{\mid \mathcal{M}_{T}}^{\omega}\right\|_{L^{2}(\mathcal{Y}) \rightarrow C(\mathcal{Y})}=\sup _{m \in \mathcal{M}_{T}} \sup _{x \in \mathcal{Y}} \frac{\left|\left(\widehat{\mathcal{T}}_{\lambda}^{*} \widehat{\mathcal{T}}_{\lambda}\right)^{\omega} m(x)\right|}{\|m\|_{L^{2}(\mathcal{Y})}}=O_{p}(1),
$$

where $0<\omega \leq 1$.

Proof. By applying Uniform Boundedness Principle (or, the Banach-Steinhaus principle), the assertion follows, if we show that, for each $m_{T} \in \mathcal{M}_{T} \subset L^{2}(\mathcal{Y})$ with $\|m\|_{L^{2}(\mathcal{Y})}=1$,

$$
\sup _{x \in \mathcal{Y}}\left|\left(\widehat{\mathcal{T}}_{\lambda}^{*} \widehat{\mathcal{T}}_{\lambda}\right)^{\omega} m_{T}(x)\right|=O_{p}(1) .
$$

Observing that $Q_{Y, \lambda}$ is symmetric nonnegative semi-definite, we can show that, for $m_{T}(\cdot) \in \mathcal{M}_{T}(\mathcal{Y})$,

$$
\begin{aligned}
\sup _{x \in \mathcal{Y}}\left|\left(\widehat{\mathcal{T}}_{\lambda}^{*} \widehat{\mathcal{T}}_{\lambda}\right)_{\mid \mathcal{M}_{T}}^{\omega} m_{T}(x)\right| & =\sup _{x \in \mathcal{Y}}\left|T^{*-2} K_{T}^{Y}(x)^{\top} M_{\lambda}^{1 / 2} Q_{Y, \lambda}^{\omega} M_{\lambda}^{-1 / 2} b_{T}\right| \\
& \leq C \sup _{x \in \mathcal{Y}}\left|T^{*-2} K_{T}^{Y}(x)^{\top} M_{\lambda}^{1 / 2} Q_{Y, \lambda} M_{\lambda}^{-1 / 2} b_{T}\right| \\
& =C \sup _{x \in \mathcal{Y}}\left|\left(\widehat{\mathcal{T}}_{\lambda}^{*} \widehat{\mathcal{T}}_{\lambda}\right)_{\mid \mathcal{M}_{T}} m_{T}(x)\right|, \text { for all } T .
\end{aligned}
$$

By the Cauchy-Schwarz (CS) inequality and boundedness of $f_{k}(\cdot, \cdot)$, it holds that

$$
\begin{aligned}
& \sup _{x}\left|\left(\widehat{\mathcal{T}}_{\lambda}^{*} \widehat{\mathcal{T}}_{\lambda}-\mathcal{T}_{\lambda}^{*} \mathcal{T}_{\lambda}\right) m_{T}(x)\right| \\
= & \sup _{x}\left|\int_{\mathcal{Y}} \int_{\mathcal{Y}} \sum_{k=2}^{\tau} \sum_{l=2}^{\tau} \lambda_{k} \lambda_{l}\left[\widehat{f}_{k}(x, w) \widehat{f_{l}}(z, w)-f_{k}(x, w) f_{l}(z, w)\right] m_{T}(z) d w d z\right| \\
& +\sum_{k=\tau+1}^{\infty} \sum_{l=\tau+1}^{\infty} \lambda_{k} \lambda_{l} \int_{\mathcal{Y}} \int_{\mathcal{Y}}\left|f_{k}(x, w) f_{l}(z, w) m_{T}(z)\right| d w d z \\
\leq & \sup _{x} \sum_{k=2}^{\tau} \sum_{l=2}^{\tau} \lambda_{k} \lambda_{l}|| \widehat{f}_{k}(x, \cdot) \widehat{f}_{l}(\cdot, \cdot)-f_{k}(x, \cdot) f_{l}(\cdot, \cdot)||_{L^{2}(\mathcal{Y}) \times L^{2}(\mathcal{Y})}|| m_{T} \|_{L^{2}(\mathcal{Y})}+C \sum_{k=\tau+1}^{\infty} \sum_{l=\tau+1}^{\infty} \lambda_{k} \lambda_{l} \\
\leq & C \sup _{x, z, w} \sum_{k=2}^{\tau} \sum_{l=2}^{\tau} \lambda_{k} \lambda_{l}\left|\widehat{f}_{k}(x, w) \widehat{f}_{l}(z, w)-f_{k}(x, w) f_{l}(z, w)\right|+o(1)=o_{p}(1),
\end{aligned}
$$

where the last inequality comes from the compact support assumption, uniform consistency of the kernel density estimate $\widehat{f}_{k}(\cdot, \cdot)$, and condition C.6. In a similar way, we can show, by the CS inequality 
and boundedness of $f_{k}(\cdot, \cdot)$, that, for any $m \in L^{2}(\mathcal{Y})$,

$$
\begin{aligned}
\sup _{x}\left|\mathcal{T}_{\lambda}^{*} \mathcal{T}_{\lambda} m(x)\right| & \leq \sup _{x} \sum_{k=2}^{\infty} \sum_{l=2}^{\infty} \lambda_{k} \lambda_{l}|| f_{k}(x, \cdot) f_{l}(\cdot, \cdot)||_{L^{2}(\mathcal{Y}) \times L^{2}(\mathcal{Y})}|| m_{T} \|_{L^{2}(\mathcal{Y})} \\
& \leq C \sup _{x, z, w} \sum_{k=2}^{\infty} \sum_{l=2}^{\infty} \lambda_{k} \lambda_{l}\left|f_{k}(x, w) f_{l}(z, w)\right|<\infty
\end{aligned}
$$

Hence, it follows that

$$
\sup _{x}\left|\widehat{\mathcal{T}}_{\lambda}^{*} \widehat{\mathcal{T}}_{\lambda} m_{T}(x)\right| \leq C \sup _{x}\left|\mathcal{T}_{\lambda}^{*} \mathcal{T}_{\lambda} m_{T}(x)\right|<\infty
$$

implying that

$$
\sup _{x \in \mathcal{Y}}\left|\left(\widehat{\mathcal{T}}_{\lambda}^{*} \widehat{\mathcal{T}}_{\lambda}\right)^{\omega} m_{T}(x)\right|<\infty
$$

as required.

Proof of Theorem 5.2. (i) We use the following error decomposition

$$
\widehat{m}_{\alpha}-m_{0}=U_{\alpha}\left(\widehat{\mathcal{T}}_{\lambda}^{*} \widehat{\mathcal{T}}_{\lambda}\right) \widehat{\mathcal{T}}_{\lambda}^{*}\left(\widehat{h}_{\lambda}-\widehat{\mathcal{T}}_{\lambda} m_{0}\right)+\left[U_{\alpha}\left(\widehat{\mathcal{T}}_{\lambda}^{*} \widehat{\mathcal{T}}_{\lambda}\right) \widehat{\mathcal{T}}_{\lambda}^{*} \widehat{\mathcal{T}}_{\lambda}-I\right] m_{0}
$$

By Proposition 5.1(i), $\widehat{\mathcal{T}}_{\lambda}: L^{2}(\mathcal{Y}) \rightarrow L^{2}(\mathcal{Y})$ is a consistent estimator for the true operator $\mathcal{T}_{\lambda}:$ $L^{2}(\mathcal{X}) \rightarrow L^{2}(\mathcal{W})$ which is bounded and one-to-one. Also, under the bandwidth conditions in C.5, it holds by Proposition 5.1(iii) that $\left\|\widehat{h}_{\lambda}-\widehat{\mathcal{T}}_{\lambda} m_{0}\right\|_{L^{2}(\mathcal{Y})} / \sqrt{\alpha} \stackrel{p}{\rightarrow} 0$, as $T \rightarrow \infty$. Now, the assertion follows, by Lemma A.2(ii) and (iii) to (20).

(ii) Let $m_{1}=\left(\mathcal{T}_{\lambda}^{*} \mathcal{T}_{\lambda}\right)^{-1} m_{0}$. The error decomposition in this case takes form of

$$
\begin{aligned}
& U_{\alpha}\left(\widehat{\mathcal{T}}_{\lambda}^{*} \widehat{\mathcal{T}}_{\lambda}\right) \widehat{\mathcal{T}}_{\lambda}^{*}\left(\widehat{h}_{\lambda}-\widehat{\mathcal{T}}_{\lambda} m_{0}\right)+\left[\left(U_{\alpha}\left(\widehat{\mathcal{T}}_{\lambda}^{*} \widehat{\mathcal{T}}_{\lambda}\right) \widehat{\mathcal{T}}_{\lambda}^{*} \widehat{\mathcal{T}}_{\lambda}-I\right) \widehat{\mathcal{T}}_{\lambda}^{*} \widehat{\mathcal{T}}_{\lambda}\right] m_{1} \\
& -\left[\left(U_{\alpha}\left(\widehat{\mathcal{T}}_{\lambda}^{*} \widehat{\mathcal{T}}_{\lambda}\right) \widehat{\mathcal{T}}_{\lambda}^{*} \widehat{\mathcal{T}}_{\lambda}-I\right) \widehat{\mathcal{T}}_{\lambda}^{*}\right]\left(\widehat{\mathcal{T}}_{\lambda}-\mathcal{T}_{\lambda}\right) m_{1}-\left(U_{\alpha}\left(\widehat{\mathcal{T}}_{\lambda}^{*} \widehat{\mathcal{T}}_{\lambda}\right) \widehat{\mathcal{T}}_{\lambda}^{*} \widehat{\mathcal{T}}_{\lambda}-I\right)\left(\widehat{\mathcal{T}}_{\lambda}^{*}-\mathcal{T}_{\lambda}^{*}\right) \mathcal{T}_{\lambda} m_{1}
\end{aligned}
$$

By Lemma A.2(ii), $L^{2}$-norm of the first term is bounded by $\frac{C_{1}}{\sqrt{\alpha}}\left\|\widehat{h}_{\lambda}-\widehat{\mathcal{T}}_{\lambda} m_{0}\right\|_{L^{2}(\mathcal{W})}$, almost surely. By applying Lemma A.3(i) and (ii), we get:

$$
\begin{aligned}
\left\|\left(U_{\alpha}\left(\widehat{\mathcal{T}}_{\lambda}^{*} \widehat{\mathcal{T}}_{\lambda}\right) \widehat{\mathcal{T}}_{\lambda}^{*} \widehat{\mathcal{T}}_{\lambda}-I\right) \widehat{\mathcal{T}}_{\lambda}^{*} \widehat{\mathcal{T}}_{\lambda}\right\| & \leq C \alpha, \text { a.s } \\
\left\|\left(U_{\alpha}\left(\widehat{\mathcal{T}}_{\lambda}^{*} \widehat{\mathcal{T}}_{\lambda}\right) \widehat{\mathcal{T}}_{\lambda}^{*} \widehat{\mathcal{T}}_{\lambda}-I\right) \widehat{\mathcal{T}}_{\lambda}^{*}\right\| & \leq C \alpha^{1 / 2}, \text { a.s. }
\end{aligned}
$$

Also, by A.2.(iii), it holds that $\left(U_{\alpha}\left(\widehat{\mathcal{T}}_{\lambda}^{*} \widehat{\mathcal{T}}_{\lambda}\right) \widehat{\mathcal{T}}_{\lambda}^{*} \widehat{\mathcal{T}}_{\lambda}-I\right) \leq C$, which, together with the results of Proposition 5.1, completes the proof. 
Proof of Theorem 5.3 Let $m_{1}=\left(\mathcal{T}_{\lambda}^{*} \mathcal{T}_{\lambda}\right)^{-1} m_{0}$. From the proof of Theorem 5.2(ii),

$$
\begin{aligned}
& U_{\alpha}\left(\widehat{\mathcal{T}}_{\lambda}^{*} \widehat{\mathcal{T}}_{\lambda}\right) \widehat{\mathcal{T}}_{\lambda}^{*}\left(\widehat{h}_{\lambda}-\widehat{\mathcal{T}}_{\lambda} m_{0}\right)+\left[U_{\alpha}\left(\widehat{\mathcal{T}}_{\lambda}^{*} \widehat{\mathcal{T}}_{\lambda}\right) \widehat{\mathcal{T}}_{\lambda}^{*} \widehat{\mathcal{T}}_{\lambda}-I\right] \widehat{\mathcal{T}}_{\lambda}^{*} \widehat{\mathcal{T}}_{\lambda} m_{1} \\
& -\left[U_{\alpha}\left(\widehat{\mathcal{T}}_{\lambda}^{*} \widehat{\mathcal{T}}_{\lambda}\right) \widehat{\mathcal{T}}_{\lambda}^{*} \widehat{\mathcal{T}}_{\lambda}-I\right] \widehat{\mathcal{T}}_{\lambda}^{*}\left(\widehat{\mathcal{T}}_{\lambda}-\mathcal{T}_{\lambda}\right) m_{1} \\
& -U_{\alpha}\left(\widehat{\mathcal{T}}_{\lambda}^{*} \widehat{\mathcal{T}}_{\lambda}\right) \widehat{\mathcal{T}}_{\lambda}^{*} \widehat{\mathcal{T}}_{\lambda}\left(\widehat{\mathcal{T}}_{\lambda}^{*}-\mathcal{T}_{\lambda}^{*}\right) \mathcal{T}_{\lambda} m_{1}+\left(\widehat{\mathcal{T}}_{\lambda}^{*}-\mathcal{T}_{\lambda}^{*}\right) \mathcal{T}_{\lambda} m_{1} \\
& \\
& \xi_{1 T}+\xi_{2 T}+\xi_{3 T}+\xi_{4 T}+\xi_{5 T} .
\end{aligned}
$$

Let $\omega>0$ be any fixed real number. In the proof of Theorem 4.2 (step II), we showed the spectral representation of $r\left(\widehat{\mathcal{T}}_{\lambda}^{*} \widehat{\mathcal{T}}_{\lambda}\right)$ from which follows

$$
\left(\widehat{\mathcal{T}}_{\lambda}^{*} \widehat{\mathcal{T}}_{\lambda}\right)^{\omega} m(x)=T^{*-2} K_{T}^{Y}(x)^{\top} M_{\lambda}^{1 / 2} Q_{Y, \lambda}^{\omega-1} M_{\lambda}^{1 / 2}<K_{T}^{Y}, m>_{L^{2}(\mathcal{Y})} .
$$

Since, for each $T,\left(\widehat{\mathcal{T}}_{\lambda}^{*} \widehat{\mathcal{T}}_{\lambda}\right)^{\omega} m$ is a continuous function for any $m \in L^{2}(\mathcal{Y})$, it holds that

$$
\mathcal{R}\left[\left(\widehat{\mathcal{T}}_{\lambda}^{*} \widehat{\mathcal{T}}_{\lambda}\right)^{\omega}\right] \subset C(\mathcal{Y}), \text { for each } T
$$

i.e., $\left(\widehat{\mathcal{T}}_{\lambda}^{*} \widehat{\mathcal{T}}_{\lambda}\right)^{\omega}: L^{2}(\mathcal{Y}) \rightarrow C(\mathcal{Y})$ is well defined. Let $\mathcal{M}_{T}(\mathcal{Y})=\left\{m \in L^{2}(\mathcal{Y}): m_{T}(x)=T^{-2} K_{T}^{Y}(x)^{\top} b_{T}\right.$, for $\left.b_{T} \in \mathbb{R}^{T}\right\}$. Define $\left(\widehat{\mathcal{T}}_{\lambda}^{*} \widehat{\mathcal{T}}_{\lambda}\right)_{\mid \mathcal{M}_{T}}^{\omega}: \mathcal{M}_{T} \rightarrow C(\mathcal{Y})$. Note that, under the given conditions, $\widehat{f}_{k}(\cdot, \cdot)$ is the uniformly consistent estimate of $f_{k}(\cdot, \cdot)$. Consequently, by Lemma A.4, $\left(\widehat{\mathcal{T}}_{\lambda}^{*} \widehat{\mathcal{T}}_{\lambda}\right)_{\mid \mathcal{M}_{T}}^{\omega}: \mathcal{M}_{T} \rightarrow C(\mathcal{Y})$ is uniformly bounded in the sense that

$$
\|\left(\widehat{\mathcal{T}}_{\lambda}^{*} \widehat{\mathcal{T}}_{\lambda}\right)_{\mid \mathcal{M}_{T}}^{\omega}||_{L^{2}(\mathcal{Y}) \rightarrow C(\mathcal{Y})}=\sup _{m \in \mathcal{M}_{T}} \sup _{x \in \mathcal{Y}} \frac{\left|\widehat{\mathcal{T}}_{\lambda}^{*} \widehat{\mathcal{T}}_{\lambda} m(x)\right|}{\left.|| m\right|_{L^{2}(\mathcal{Y})}}=O_{p}(1) .
$$

From the definition of the operator norm and $\mathcal{R}\left\{U_{\alpha}\left(\widehat{\mathcal{T}}_{\lambda}^{*} \widehat{\mathcal{T}}_{\lambda}\right)\left(\widehat{\mathcal{T}}_{\lambda}^{*} \widehat{\mathcal{T}}_{\lambda}\right)^{-\omega} \widehat{\mathcal{T}}_{\lambda}^{*}\right\} \subset \mathcal{M}_{T}$, we obtain

$$
\begin{aligned}
& \sup _{x \in \mathcal{Y}}\left|\xi_{1 T}(x)\right| \\
= & \sup _{x \in \mathcal{Y}}\left|U_{\alpha}\left(\widehat{\mathcal{T}}_{\lambda}^{*} \widehat{\mathcal{T}}_{\lambda}\right) \widehat{\mathcal{T}}_{\lambda}^{*}\left(\widehat{h}_{\lambda}-\widehat{\mathcal{T}}_{\lambda} m_{0}\right)(x)\right| \\
= & \sup _{x \in \mathcal{Y}}\left|\left(\widehat{\mathcal{T}}_{\lambda}^{*} \widehat{\mathcal{T}}_{\lambda}\right)^{\omega}\left[U_{\alpha}\left(\widehat{\mathcal{T}}_{\lambda}^{*} \widehat{\mathcal{T}}_{\lambda}\right)\left(\widehat{\mathcal{T}}_{\lambda}^{*} \widehat{\mathcal{T}}_{\lambda}\right)^{-\omega} \widehat{\mathcal{T}}_{\lambda}^{*}\left(\widehat{h}_{\lambda}-\widehat{\mathcal{T}}_{\lambda} m_{0}\right)\right](x)\right| \\
\leq & \left\|\left(\widehat{\mathcal{T}}_{\lambda}^{*} \widehat{\mathcal{T}}_{\lambda}\right)_{\mid \mathcal{M}_{T}}^{\omega}||_{L^{2}(\mathcal{Y}) \rightarrow C(\mathcal{Y})}|| U_{\alpha}\left(\widehat{\mathcal{T}}_{\lambda}^{*} \widehat{\mathcal{T}}_{\lambda}\right)\left(\widehat{\mathcal{T}}_{\lambda}^{*} \widehat{\mathcal{T}}_{\lambda}\right)^{-\omega} \widehat{\mathcal{T}}_{\lambda}^{*}||_{L^{2}(\mathcal{Y}) \rightarrow L^{2}(\mathcal{Y})}\left|\widehat{h}_{\lambda}-\widehat{\mathcal{T}}_{\lambda} m_{0} \|\right|_{L^{2}(\mathcal{Y})}\right. \\
\leq & C \alpha^{-1 / 2-2 \omega}|| \widehat{h}_{\lambda}-\widehat{\mathcal{T}}_{\lambda} m_{0}||_{L^{2}(\mathcal{Y})}=O_{p}\left(\alpha^{-1 / 2-2 \omega}\left[1 / \sqrt{T g_{2}}+g_{1}^{p_{0}}\right]\right), \text { for any } \omega>0,
\end{aligned}
$$

where the last equality comes from Proposition 5.1. Since we assume that $\alpha=o\left(1 /(\log T)^{c}\right)$, for any $c>0$, we get, by letting $c=1 / 2 \omega$,

$$
\sup _{x \in \mathcal{Y}}\left|\xi_{1 T}(x)\right|=O_{p}\left(\alpha^{-1 / 2}\left[1 / \sqrt{T g_{2}}+g_{1}^{p_{0}}\right] \log T\right) .
$$


By the same arguments, we have

$$
\begin{aligned}
& \sup _{x \in \mathcal{Y}}\left|\xi_{4 T}(x)\right|=\sup _{x \in \mathcal{Y}}\left|\left(\widehat{\mathcal{T}}_{\lambda}^{*} \widehat{\mathcal{T}}_{\lambda}\right)^{\omega}\left[U_{\alpha}\left(\widehat{\mathcal{T}}_{\lambda}^{*} \widehat{\mathcal{T}}_{\lambda}\right)\left(\widehat{\mathcal{T}}_{\lambda}^{*} \widehat{\mathcal{T}}_{\lambda}\right)^{1-\omega}\left(\widehat{\mathcal{T}}_{\lambda}^{*}-\mathcal{T}_{\lambda}^{*}\right) \mathcal{T}_{\lambda} m_{1}\right](x)\right| \\
& \leq\left.\left\|\left.\left(\widehat{\mathcal{T}}_{\lambda}^{*} \widehat{\mathcal{T}}_{\lambda}\right)_{\mid \mathcal{M}_{T}}^{\omega}\right|_{L^{2}(\mathcal{Y}) \rightarrow C(\mathcal{Y})}\right\| U_{\alpha}\left(\widehat{\mathcal{T}}_{\lambda}^{*} \widehat{\mathcal{T}}_{\lambda}\right)\left(\widehat{\mathcal{T}}_{\lambda}^{*} \widehat{\mathcal{T}}_{\lambda}\right)^{1-\omega}\right|_{L^{2}(\mathcal{Y}) \rightarrow L^{2}(\mathcal{Y})}\left\|\left(\widehat{\mathcal{T}}_{\lambda}^{*}-\mathcal{T}_{\lambda}^{*}\right) \mathcal{T}_{\lambda} m_{1}\right\|_{L^{2}(\mathcal{Y})} \\
& =O_{p}\left(\alpha^{-\omega}\left[1 / \sqrt{T g_{1}}+g_{1}^{p_{0}}+g_{2}^{p_{0}}\right]\right)=O_{p}\left(\left[1 / \sqrt{T g_{1}}+g_{1}^{p_{0}}+g_{2}^{p_{0}}\right] \log T\right), \\
& \sup _{x \in \mathcal{Y}}\left|\xi_{2 T}(x)\right|=\sup _{x \in \mathcal{Y}}\left|\left(\widehat{\mathcal{T}}_{\lambda}^{*} \widehat{\mathcal{T}}_{\lambda}\right)^{\omega}\left\{\left[U_{\alpha}\left(\widehat{\mathcal{T}}_{\lambda}^{*} \widehat{\mathcal{T}}_{\lambda}\right) \widehat{\mathcal{T}}_{\lambda}^{*} \widehat{\mathcal{T}}_{\lambda}-I\right]\left(\widehat{\mathcal{T}}_{\lambda}^{*} \widehat{\mathcal{T}}_{\lambda}\right)^{1-\omega} m_{1}\right\}(x)\right| \\
& \leq\left\|\left(\widehat{\mathcal{T}}_{\lambda}^{*} \widehat{\mathcal{T}}_{\lambda}\right)_{\mid \mathcal{M}_{T}}^{\omega}\right\|_{L^{2}(\mathcal{Y}) \rightarrow C(\mathcal{Y})}\left\|\left[U_{\alpha}\left(\widehat{\mathcal{T}}_{\lambda}^{*} \widehat{\mathcal{T}}_{\lambda}\right) \widehat{\mathcal{T}}_{\lambda}^{*} \widehat{\mathcal{T}}_{\lambda}-I\right]\left(\widehat{\mathcal{T}}_{\lambda}^{*} \widehat{\mathcal{T}}_{\lambda}\right)^{1-\omega}\right\|_{L^{2}(\mathcal{Y}) \rightarrow L^{2}(\mathcal{Y})}\left\|m_{1}\right\|_{L^{2}(\mathcal{Y})} \\
& \leq C \alpha^{1-2 \omega}|| m_{1} \|_{L^{2}(\mathcal{Y})}=O_{p}(\alpha \log T) \text {, }
\end{aligned}
$$

and

$$
\begin{aligned}
\sup _{x \in \mathcal{Y}}\left|\xi_{3 T}(x)\right| & =\sup _{x \in \mathcal{Y}}\left|\left(\widehat{\mathcal{T}}_{\lambda}^{*} \widehat{\mathcal{T}}_{\lambda}\right)^{\omega}\left\{\left[U_{\alpha}\left(\widehat{\mathcal{T}}_{\lambda}^{*} \widehat{\mathcal{T}}_{\lambda}\right) \widehat{\mathcal{T}}_{\lambda}^{*} \widehat{\mathcal{T}}_{\lambda}-I\right]\left(\widehat{\mathcal{T}}_{\lambda}^{*} \widehat{\mathcal{T}}_{\lambda}\right)^{-\omega} \widehat{\mathcal{T}}_{\lambda}^{*}\left(\widehat{\mathcal{T}}_{\lambda}-\mathcal{T}_{\lambda}\right) m_{1}\right\}(x)\right| \\
& \leq\left\|\left(\widehat{\mathcal{T}}_{\lambda}^{*} \widehat{\mathcal{T}}_{\lambda}\right)_{\mid \mathcal{M}_{T}}^{\omega}||_{L^{2}(\mathcal{Y}) \rightarrow C(\mathcal{Y})}||\left[U_{\alpha}\left(\widehat{\mathcal{T}}_{\lambda}^{*} \widehat{\mathcal{T}}_{\lambda}\right) \widehat{\mathcal{T}}_{\lambda}^{*} \widehat{\mathcal{T}}_{\lambda}-I\right]\left(\widehat{\mathcal{T}}_{\lambda}^{*} \widehat{\mathcal{T}}_{\lambda}\right)^{-\omega} \widehat{\mathcal{T}}_{\lambda}^{*}||_{L^{2}(\mathcal{Y}) \rightarrow L^{2}(\mathcal{Y})}||\left(\widehat{\mathcal{T}}_{\lambda}-\mathcal{T}_{\lambda}\right) m_{1}\right\|_{L^{2}(\mathcal{Y})} \\
& \leq C \alpha^{1 / 2-2 \omega}\left\|\left(\widehat{\mathcal{T}}_{\lambda}-\mathcal{T}_{\lambda}\right) m_{1}\right\|_{L^{2}(\mathcal{Y})}=O_{p}\left(\alpha^{1 / 2}\left[1 / \sqrt{T g_{2}}+g_{1}^{p_{0}}+g_{2}^{p_{0}}\right] \log T\right)
\end{aligned}
$$

For the uniform convergence rate of $\xi_{4 T}(x)=\left(\widehat{\mathcal{T}}_{\lambda}^{*}-\mathcal{T}_{\lambda}^{*}\right) h_{1}$, we note that $\widehat{\mathcal{T}}_{\lambda}^{*} h_{1}$ is equivalent (up to some bias term) to the standard one-dimensional kernel estimate, where $h_{1}=\mathcal{T}_{\lambda} m_{1}$. Hence, its uniform convergence rate follows from application of Masry $(1996)^{4}$ :

$$
\sup _{x \in \mathcal{Y}}\left|\xi_{3 T}(x)\right|=O_{p}\left(\left[1 / \sqrt{T g_{1}}+g_{1}^{p_{0}}+g_{2}^{p_{0}}\right] \log T\right) .
$$

Proof of Theorem 5.4. First, we show that $\widehat{\beta}$ is consistent, which follows from the consistency of $\widetilde{\beta}$ and the uniform approximation of $\widehat{\ell}(\beta)$ by $\ell(\beta)$ over $B$. Then, we establish that $\widehat{\ell}(\beta)-\ell(\beta)=$ $o_{p}\left(T^{-1 / 4}\right)$ uniformly over a shrinking neighborhood of $\beta_{0}$. The argument is fairly standard so we just sketch the second part here. We have

$$
|\widehat{\ell}(\beta)-\ell(\beta)| \leq \frac{1}{T} \sum_{t=1}^{T}\left|\ln \frac{\sigma_{t}^{2}\left(\beta, \widehat{m}_{\alpha}\right)}{\sigma_{t}^{2}\left(\beta, m_{0}\right)}\right|+\frac{1}{T} \sum_{t=1}^{T} y_{t}^{2} \frac{\left|\sigma_{t}^{2}\left(\beta, \widehat{m}_{\alpha}\right)-\sigma_{t}^{2}\left(\beta, m_{0}\right)\right|}{\sigma_{t}^{2}\left(\beta, m_{0}\right) \sigma_{t}^{2}\left(\beta, \widehat{m}_{\alpha}\right)}
$$

where

$$
\begin{aligned}
\max _{\tau_{\dagger} \leq t \leq T}\left|\sigma_{t}^{2}\left(\beta, \widehat{m}_{\alpha}\right)-\sigma_{t}^{2}\left(\beta, m_{0}\right)\right| \leq & \sum_{j=1}^{\infty} \beta^{j-1} \sup _{y \in \mathcal{Y}}\left|\widehat{m}_{\alpha}(y)-m_{0}(y)\right| \\
& +\sum_{j=\tau_{\dagger}+1}^{\infty} \beta^{j-1}\left[(1-\beta) \max _{\tau_{\dagger} \leq t \leq T} y_{t-j}^{2}+\max _{\tau_{\dagger} \leq t \leq T} m\left(y_{t-j}\right)\right] \\
= & o_{p}\left(T^{-1 / 4}\right),
\end{aligned}
$$

\footnotetext{
${ }^{4}$ More rigorously, we may follow the same line of the proof of Proposition 3.1(ii) to apply the uniform convergence results in Masry (1996).
} 
since $y_{s}$ and $m\left(y_{s}\right)$ are bounded by assumption. Using $\ln (1+\delta) \leq|\delta|$ for $|\delta|<1$, we can bound the first term in (21) with probability tending to one by

$$
\left|\frac{1}{T} \sum_{t=1}^{T} \ln \frac{\sigma_{t}^{2}\left(\beta, \widehat{m}_{\alpha}\right)}{\sigma_{t}^{2}\left(\beta, m_{0}\right)}\right| \leq \frac{1}{T} \sum_{t=1}^{T}\left|\frac{\sigma_{t}^{2}\left(\beta, \widehat{m}_{\alpha}\right)-\sigma_{t}^{2}\left(\beta, m_{0}\right)}{\sigma_{t}^{2}\left(\beta, m_{0}\right)}\right|=o_{p}\left(T^{-1 / 4}\right),
$$

since $\min _{\tau_{\dagger} \leq t \leq T} \sigma_{t}^{2}\left(\beta, m_{0}\right)$ is bounded away from zero. Likewise the second term in $(21)$ is $o_{p}\left(T^{-1 / 4}\right)$.

\section{References}

[1] Ai, C. And X. Chen (2003): "Efficient estimation of models with conditional moment restrictions containing unknown functions," Econometrica 71(6), 1795-1844.

[2] Birman, M. And M. Solomjak (1980): Quantitative Analysis in Sobolev Imbedding Theorems and Applications to Spectral Theory. American Math. Soc. Tran., series 2, Vol 114.

[3] Blundell, R., X. Chen, and D. Kristensen (2004): "Semiparametric Estimation of Shape Invariant Engle Curves under Endogeneity" CEMMAP working paper WP15/03

[4] Blundell, R. And J. L. Powell (2001a): "Endogeneity in nonparametric and semiparametric regression models," forthcoming in Advances in Econometrics, Proceedings of the World Meetings, 2000, ed. by L. Hansen, North Holland.

[5] Blundell, R. And J. L. Powell (2001b): "Endogeneity in semiparametric binary response models," Center for Microdata Methods and Practice, Working Paper, 05/01.

[6] Carrasco, M., J.P. Florens, and E. Renault (2006), "Linear Inverse problems in Structural Econometrics," Handbook of Econometrics, volume 6, eds. J.J. Heckman and E. Leamer.

[7] Chen, X. and X. Shen (1998). Sieve extremum estimates for weakly dependent data. Econometrica 66, 289-314.

[8] Darolles, S., J-P. Florens, And E. Renault (2001): "Nonparametric instrumental regression, Working Paper, GREMAQ, University of Toulouse.

[9] DAs, M. (1999): "Instrumental variable estimation of models with discrete endogenous regressors, " presented at 2000 World Congress of the Econometric Society.

[10] ENGL, H. (1987): "On the choice of the regularization parameter for iterated Tikhonov regularization of ill-posed problems," Journal of Approximation Theory 49, 55-63. 
[11] Engl, H. And H. GFrerer (1988): "A posteriori parameter choice for general regularization methods for solving linear ill-posed problems," Applied Numerical Mathematics 4, 395-417.

[12] Engle, R.F., And T. Bollerslev (1986): "Modelling the persistence of Conditional Variances," Econometric Reviews 5, 1-50.

[13] Engle, H.W., M. Hanke, And A. Neubauer (2000): Regularization of Inverse Problems. Dordrecht: Kluwer Academic Press.

[14] FAn, J. (1991): "Global behavior of deconcovolution kernel estimates," Statistica Sinica 1, 541551.

[15] Groetsch, C.W. (1993): Inverse Problems in the Mathematical Sciences. Braunschweig: Vieweg.

[16] Groetsch, C.W. (1983): "On the Asymptotic Order of Accuracy of Tikhonov Regularization," Journal of Optimization Theory and Applications 41, 293-298.

[17] Groetsch, C.W. (1977): Generalized Inverses of Linear Operators: Representation and Approximation. New York: Dekker.

[18] Hall P. And C.C. Heyde (1980): Martingale Limit Theory andIts Applications. Academic Press, New York.

[19] Hall P. And J. L. Horowitz (2005): "Nonparametric methods for inference in the presence of instrumental variables," The Annals of Statistics .

[20] Hansen L. P. (1982): "Large sample properties of generalized method of moments estimators," Econometrica 50, 1029-1054.

[21] Harvey, A., E. Ruiz, And N. Shephard (1994): "Multivariate Stochastic Variance Models," Review of Economic Studies 61, 247-64.

[22] HiLl, B. M. (1975), A Simple General Approach to Inference about the Tail of a Distribution, Annals of Statistics 3, 1163-1174.

[23] Hutson, V. And J. S. Pym (1980): Applications of Functional Analysis and Operator Theory. London: Academic Press.

[24] Imbens, G. W. And W. K. Newey (2001): "Identification and estimation of triangular simultaneous equations models without additivity, Working Paper. 
[25] Ivanov, V.K., V.V. Vasin, and V.P. Tanana (1978): Theory of Linear Ill-Posed Problems. Moscow: Nauka.

[26] KIm, W. (2003): "Identification and Estimation of Nonparametric Structural models by Instrumental Variables Method," Manuscript, Humboldt University. Available at http://ideas.repec.org/p/ecm/feam04/733.html.

[27] King, J.T. And D. Chillingworth (1979): "Approximation of generalized inverses by iterated regularization," Numerical Functional Analyses and Optimizations 1, 499-513.

[28] KIRsch, A. (1996): An Introduction to the Mathematical Theory of Inverse Problems. New York: Springer Verlag.

[29] Kress, R. (1989): Linear Integral Equations. Berlin: Springer Verlag.

[30] Lee, S., And Hansen, B. (1994): "Asymptotic Theory for the GARCH(1,1) Quasi-Maximum Likelihood Estimator," Econometric Theory, 10, 29-52.

[31] Linton, O. B. And E. Mammen (2005): "Estimating semiparametric ARCH( $\infty)$ models by kernel smoothing methods," Econometrica 73, 771-836.

[32] Mammen, E., O. B. Linton, And J. Nielsen (1999): "The existence and asymptotic properties of a backfitting projection algorithm under weak conditions," The Annals of Statistics, $27(5), 1443-1490$.

[33] MasRy, E. (1996), "Multivariate local polynomial regression for time series: Uniform strong consistency and rates," Journal of Time Series Analysis 17, 571-599.

[34] Meitz, M. And P. Saikkonen (2004). Ergodicity, mixing, and existence of moments for a class of Markov models with applications to GARCH and ACD models. Manuscript, Stockholm School of Economics.

[35] Nashed, M.Z. (1976): Generalized Inverses and Applications. New York: Academic Press.

[36] Nashed, M.Z. And G. Wahba (1974): "Convergence rates of approximate least squares solutions of linear integral and operator equations of the first kind," Mathematics of Computation $28(125), 69-80$.

[37] Nelson, D. (1990). Stationarity and persistence in the GARCH(1,1) models. Econometric Theory $6,318-334$. 
[38] Newey W. K. And J. L. Powell (1988): "Nonparametric instrumental variables estimation," MIT Working Paper.

[39] Newey W. K. AND J. L. Powell (2003): "Instrumental variable estimation of nonparametric models," Econometrica 71(5), 1565-1578.

[40] Newey W. K., J. L. Powell, And F. Vella (1999): "Nonparametric estimation of triangular simultaneous equations models," Econometrica 67, 565-603.

[41] NyChKA, D. AND D. Cox (1989): "Convergence rates for regularized solutions of integral equations from discrete noisy data," The Annals of Statistics, 17(5), 556-572.

[42] O’Sullivan, F. (1986): "Ill-posed inverse problems (with Discussion)," Statistical Science, 4, 503-527.

[43] Phillips, D.L. (1962): "A technique for the numerical solution of certain integral equations of the first kind," Journal of the Association for Computing Machinery 9, 84-97.

[44] Plato, R. and G. Vainikko (1990): "On the regularization of projection methods for solving ill-posed problems," Numerische Mathematik 57, 63-79.

[45] Rahbek, A. And S.T. Jensen (2004): "Asymptotic normality for Nonstationary, Explosive GARCH ," Econometric Theory 20, 1203-1226.

[46] Roenrig, C. S. (1988): "Conditions for Identification in Nonparametric and Parametric Models," Econometrica, 55, 875-891.

[47] SARGan, J. D. (1958): "The estimation of economic relationships using instrumental variables," Econometrica 26, 393-415.

[48] Schock, E. (1985): "Approximate solution of ill-posed equations: Arbitrarily slow convergence vs superconvergence, in Constructive Methods for the Practical Treatment of Integral Equations (edited by G. Hämmerlin and K.H. Hoffmann), pp. 234-243, Basel: Birkhäuser.

[49] Showalter D. (1967): "Representation and computation of the pseudoinverse," Proc. Amer. Math. Soc. 18, 584-586.

[50] Silverman, B.W. (1978): "Weak and strong uniform consistency of the kernel estimate of a density and its derivatives," Annals of Statistics Vol.6, No.1, 177-184.

[51] Tautenhahn, U. (1998): "Optimality for ill-posed problems under general source conditions," Numerical Functional Analyses and Optimizations 19, 37r7-398. 
[52] Theil, H. (1953): Repeated Least Squares Applied to Complete Equation Systems, The Hague; Central Planning Bureau.

[53] Tikhonov, A.N. and V. Arsenin (1977): Solutions of Ill-Posed Problems. New York: Wiley.

[54] Tikhonov, A.N (1963): "Solution of incorrectly formulated problems and the regularization method," Soviet Mathematics Doklady 4, 1035-1038.

[55] Vainikko, G.M. and A.Y. Veretennikov (1986): Iteration Procedures in Ill-Posed problems. 1st Ed. Moscow: Nauka.

[56] van Rooij, A.C.M., and F.H. Ruymgaart (1999): "On inverse estimation," in Asymptotics, Nonparametrics, and Time Series, ed. by S. Ghosh. New York: Marcel Dekker, 579-613. 